\title{
Locations and data for water wells of the Santa Rosa Valley, Sonoma County, California
}

By Zenon C. Valin and Robert J. McLaughlin

Open File Report 2005-1318

2005

U.S. Department of the Interior

U.S. Geological Survey 


\section{₹USGS \\ science for a changing world}

U.S. GEOLOGICAL SURVEY

Locations and data for water wells of the Santa Rosa Valley, Sonoma County, California

by Zenon C. Valin and Robert J. McLaughlin

Figure 1. A) Map showing location of Santa Rosa Valley area well database in northern California. B) Regional map showing well locations. C) Shaded relief map showing well locations with map numbers indicated in Table 1; numbers indicated in Table area of Quaternary units simplified from Wagner and Bortugno (1982).
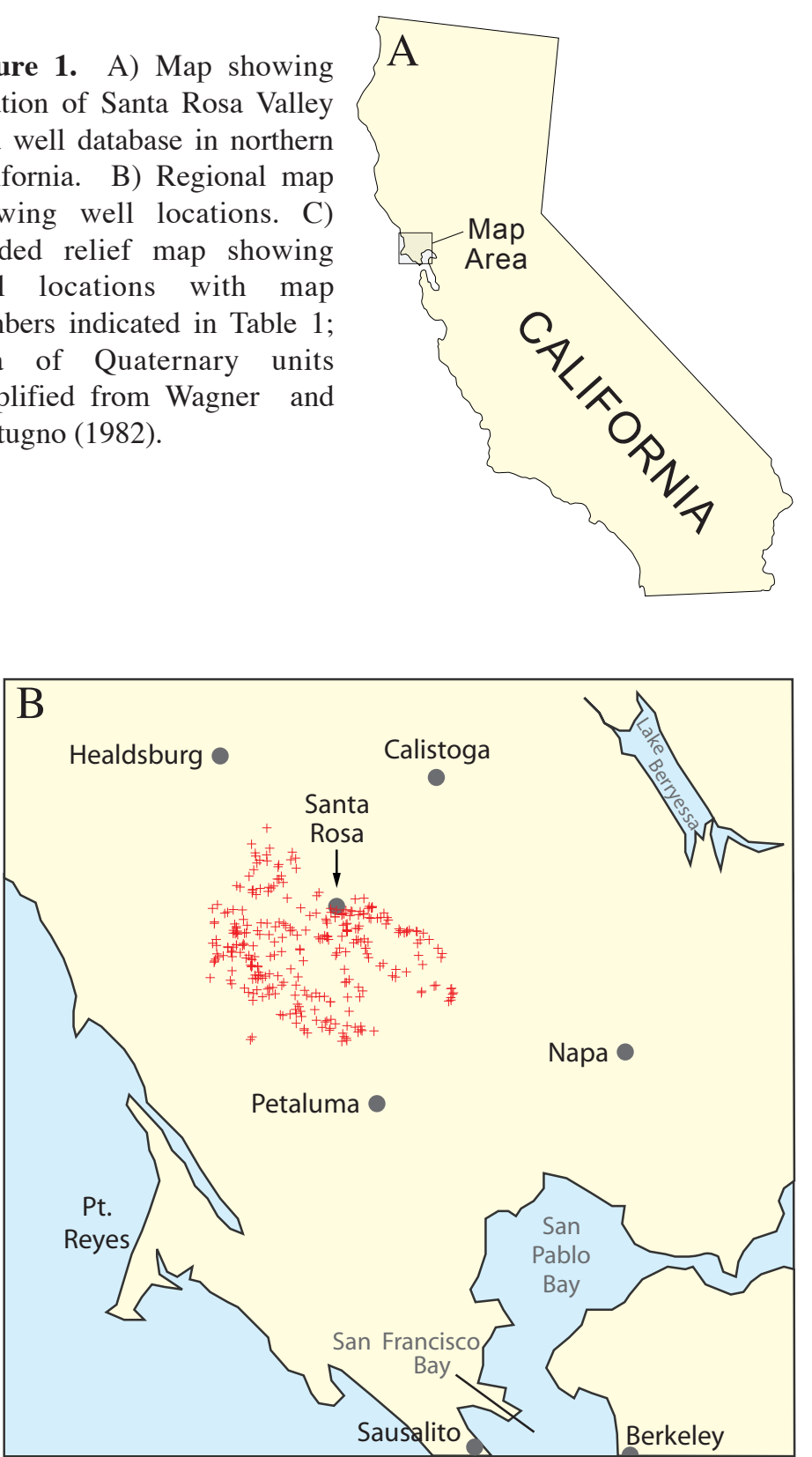

OPEN FILE REPORT 2005-1318

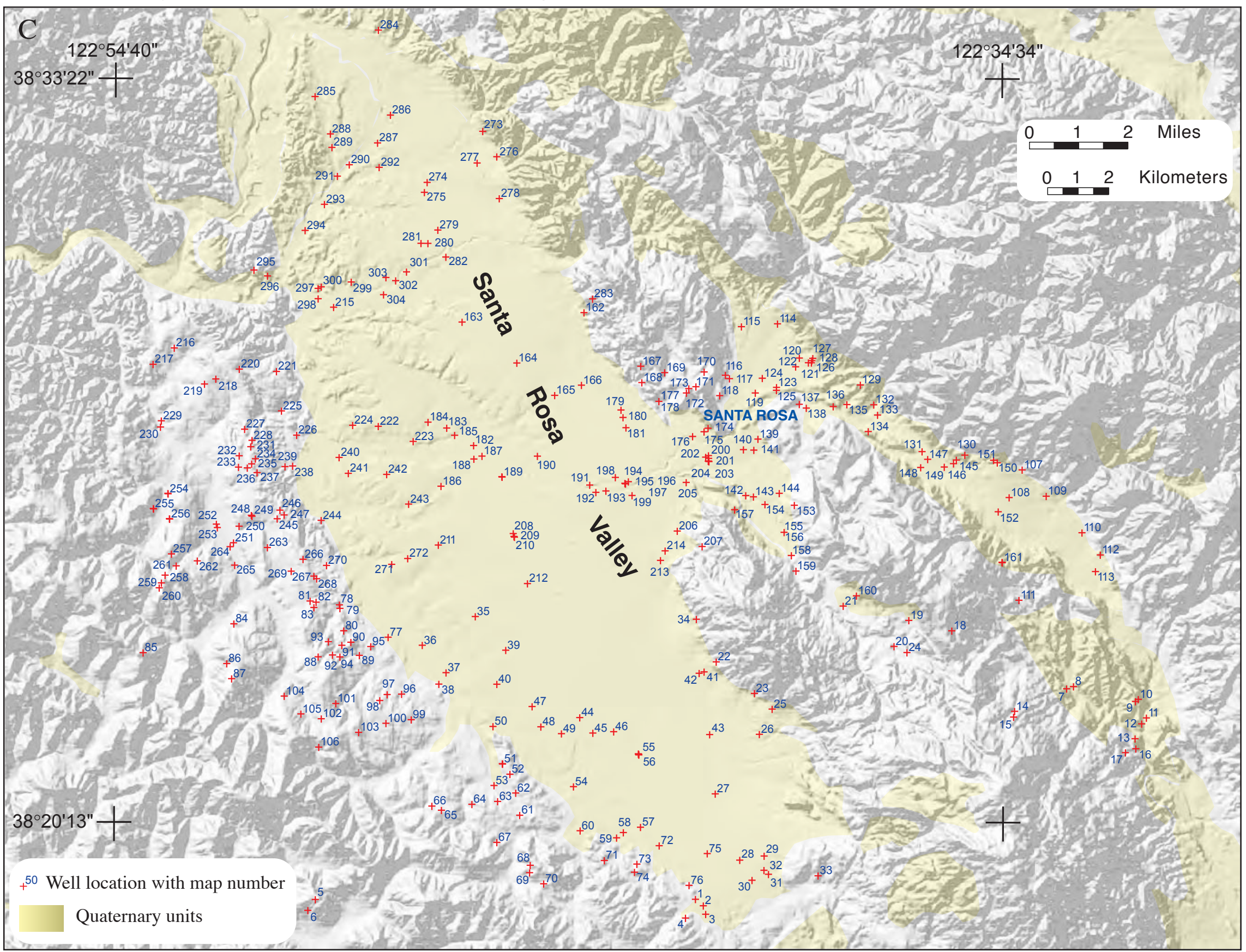


Locations and data for water wells of the Santa Rosa Valley, Sonoma County, California by Zenon C. Valin and Robert J. McLaughlin

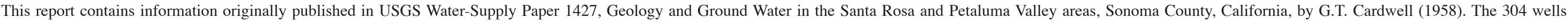

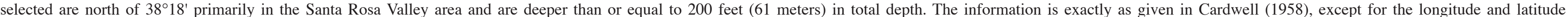

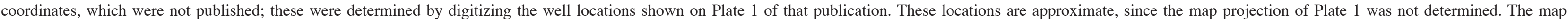
number in column 1 corresponds to the well locations in Figure 1.

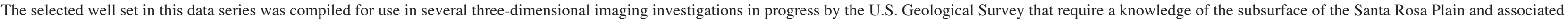

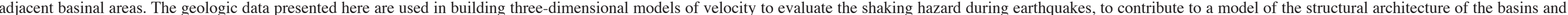
sub-basins of the area, and to assist in constructing modern hydrologic models of ground water flow. The compilation may be further used for other purposes.

Table 1. Data for selected water wells of the Santa Rosa Valley, Sonoma County, California

$[--$, no data; *, data for deepened well $]$

\begin{tabular}{|c|c|c|c|c|c|c|c|c|c|}
\hline $\begin{array}{l}\text { Map } \\
\text { Number }\end{array}$ & $\begin{array}{c}\text { Well } \\
\text { Number }\end{array}$ & $\begin{array}{c}\text { West } \\
\text { Longitude }\left({ }^{\circ}\right) \\
\end{array}$ & $\begin{array}{c}\text { North } \\
\text { Latitude }\left({ }^{\circ}\right)\end{array}$ & $\begin{array}{l}\text { Total Depth } \\
\text { (feet) }\end{array}$ & \begin{tabular}{|c|} 
Surface \\
Elevation (feet)
\end{tabular} & $\begin{array}{c}\text { Depth to top of } \\
\text { water interval (feet) }\end{array}$ & $\begin{array}{l}\text { Water interval } \\
\text { thickness (feet) }\end{array}$ & \begin{tabular}{|c|} 
Geologic unit present \\
in water interval $^{1}$
\end{tabular} & Down-Hole Lithology of water interval \\
\hline 1 & $5 / 8-1 \mathrm{~B} 1$ & 122.69191 & 38.31454 & 261 & 125 & $\begin{array}{l}129 \\
252\end{array}$ & $\begin{array}{c}39 \\
4\end{array}$ & $\begin{array}{l}\text { QTm } \\
\text { QTm }\end{array}$ & $\begin{array}{l}\text { gravel } \\
\text { gravel }\end{array}$ \\
\hline 2 & $5 / 8-1 \mathrm{H} 1$ & 122.68889 & 38.31260 & 232 & 125 & $\begin{array}{l}151 \\
224\end{array}$ & $\begin{array}{c}25 \\
6\end{array}$ & $\begin{array}{l}\text { QTm } \\
\text { QTm }\end{array}$ & $\begin{array}{l}\text { clay and gravel } \\
\text { slightly cemented sand and gravel }\end{array}$ \\
\hline 3 & $5 / 8-1 \mathrm{~J} 1$ & 122.68806 & 38.31011 & 290 & 135 & 44 & 5 & QTm & cemented gravel \\
\hline 4 & $5 / 8-1 \mathrm{~L} 1$ & 122.69568 & 38.30902 & 253 & 240 & -- & -- & QTm & -- \\
\hline 5 & 5/9-3A1 & 122.83522 & 38.31437 & 832 & 150 & -- & -- & QTm & uncased below $40 \mathrm{ft}$. \\
\hline 6 & 5/9-3G1 & 122.83794 & 38.31120 & 1010 & 85 & $\begin{array}{c}3 \\
749 \\
840 \\
956\end{array}$ & $\begin{array}{c}743 \\
3 \\
92 \\
15\end{array}$ & $\begin{array}{l}\text { QTm } \\
\text { QTm } \\
\text { QTm } \\
\text { QTm }\end{array}$ & $\begin{array}{l}\text { sandstone } \\
\text { sandstone, streaks of rock } \\
\text { clay and gravel; clay and boulders } \\
\text { coarse sandstone }\end{array}$ \\
\hline 7 & $6 / 6-8 \mathrm{~N} 1$ & 122.55190 & 38.37633 & 490 & 325 & -- & -- & QTge, Tsv(?) & -- \\
\hline 8 & 6/6-8P1 & 122.54922 & 38.37701 & 270 & 310 & -- & -- & QTge & -- \\
\hline 9 & 6/6-16B1 & 122.52593 & 38.37260 & 426 & 400 & -- & -- & QTge, Tsv(?) & -- \\
\hline 10 & 6/6-16B2 & 122.52483 & 38.37320 & 300 & 211 & -- & -- & QTge & -- \\
\hline 11 & 6/6-16H1 & 122.52185 & 38.36773 & 210 & 300 & -- & -- & QTge, Tsv(?) & -- \\
\hline 12 & $6 / 6-16 \mathrm{~J} 2$ & 122.52357 & 38.36604 & 280 & 260 & -- & -- & -- & -- \\
\hline 13 & 6/6-16Q1 & 122.52604 & 38.36166 & 360 & 235 & 260 & 100 & Tsv & shale \\
\hline 14 & 6/6-18E1 & 122.57154 & 38.36980 & 225 & 925 & 204 & 21 & Tsv & "shale" rock \\
\hline 15 & 6/6-18E2 & 122.57188 & 38.36804 & 214 & 975 & 175 & 5 & Tsv & rock \\
\hline 16 & 6/6-21B2 & 122.52583 & 38.35871 & 240 & 275 & 192 & 48 & QTge(?) & very porous volcanic rock \\
\hline 17 & 6/6-21C1 & 122.52975 & 38.35752 & 231 & 365 & -- & -- & - & -- \\
\hline 18 & $6 / 7-2 J 1$ & 122.59512 & 38.39349 & 358 & 995 & 335 & -- & Tsv & rock \\
\hline 19 & 6/7-3H1 & 122.61134 & 38.39657 & 520 & 575 & -- & -- & Qyal, Tsv & -- \\
\hline
\end{tabular}


Locations and data for water wells of the Santa Rosa Valley, Sonoma County, California

by Zenon C. Valin and Robert J. McLaughlin

Table 1. Data for selected water wells of the Santa Rosa Valley, Sonoma County, California-Continued

$[--$, no data; *, data for deepened well]

\begin{tabular}{|c|c|c|c|c|c|c|c|c|c|}
\hline $\begin{array}{l}\text { Map } \\
\text { Number }\end{array}$ & \begin{tabular}{|c|} 
Well \\
Number
\end{tabular} & $\begin{array}{c}\text { West } \\
\text { Longitude }\left(^{\circ}\right)\end{array}$ & $\begin{array}{c}\text { North } \\
\text { Latitude }\left({ }^{\circ}\right)\end{array}$ & $\begin{array}{l}\text { Total Depth } \\
\text { (feet) }\end{array}$ & \begin{tabular}{|c|} 
Surface \\
Elevation (feet)
\end{tabular} & $\begin{array}{c}\text { Depth to top of } \\
\text { water interval (feet) }\end{array}$ & $\begin{array}{l}\text { Water interval } \\
\text { thickness (feet) }\end{array}$ & $\begin{array}{c}\text { Geologic unit present } \\
\text { in water interval }\end{array}$ & Down-Hole Lithology of water interval \\
\hline 20 & $6 / 7-3 Q 1$ & 122.61694 & 38.38889 & 417 & 555 & $\begin{array}{c}27 \\
98 \\
125\end{array}$ & $\begin{array}{c}23 \\
12 \\
292\end{array}$ & $\begin{array}{c}\text { QTge } \\
\text { QTge } \\
\text { Tsv }\end{array}$ & $\begin{array}{l}\text { gravel } \\
\text { wash gravel } \\
\text { white ash }\end{array}$ \\
\hline 21 & $6 / 7-4 \mathrm{~B} 1$ & 122.63611 & 38.40086 & 609 & 440 & -- & -- & Tsv, Tp & dry hole \\
\hline 22 & 6/7-7E1 & 122.68410 & 38.38441 & 278 & 240 & -- & -- & Tsv & -- \\
\hline 23 & 6/7-7R1 & 122.66964 & 38.37511 & 265 & 205 & -- & -- & QTge(?) & -- \\
\hline 24 & 6/7-10A1 & 122.61206 & 38.38709 & 350 & 600 & -- & -- & -- & -- \\
\hline 25 & $6 / 7-17 \mathrm{E} 1$ & 122.66291 & 38.37050 & 650 & 225 & -- & -- & -- & -- \\
\hline 26 & 6/7-18R1 & 122.66777 & 38.36308 & 250 & 160 & -- & -- & -- & -- \\
\hline 27 & $6 / 7-19 \mathrm{~N} 1$ & 122.68446 & 38.34556 & 340 & 117 & -- & -- & QTge(?), QTm & -- \\
\hline 28 & 6/7-31G1 & 122.67519 & 38.32600 & 508 & 135 & -- & -- & -- & -- \\
\hline 29 & $6 / 7-31 \mathrm{H} 1$ & 122.66602 & 38.32725 & 298 & 160 & -- & -- & Qyal, QTge(?) & -- \\
\hline 30 & $6 / 7-31 \mathrm{~J} 1$ & 122.67062 & 38.32007 & 280 & 135 & $\begin{array}{c}45 \\
103 \\
170 \\
218\end{array}$ & $\begin{array}{l}49 \\
37 \\
28 \\
56\end{array}$ & $\begin{array}{l}\text { QTge(?) } \\
\text { QTge(?) } \\
\text { QTge(?) } \\
\text { QTm }\end{array}$ & $\begin{array}{l}\text { sandy clay and gravel } \\
\text { clay and gravel; cemented gravel } \\
\text { boulders; clay and gravel } \\
\text { sand; sandstone; sand and gravel }\end{array}$ \\
\hline 31 & 6/7-32M & 122.66438 & 38.32185 & 1163 & 145 & - & -- & -- & -- \\
\hline 32 & $6 / 7-32 \mathrm{M} 2$ & 122.66604 & 38.32304 & 200 & 150 & 190 & 10 & -- & coarse sand \\
\hline 33 & 6/7-33M1 & 122.64565 & 38.32139 & 317 & 185 & 294 & 21 & $\mathrm{Tp}(?)$ & cemented gravel; gravel \\
\hline 34 & 6/8-1G1 & 122.69152 & 38.39693 & 276 & 225 & - & -- & Tsv & -- \\
\hline 35 & $6 / 8-5 \mathrm{E} 1$ & 122.77492 & 38.39777 & 638 & 85 & $\begin{array}{c}0 \\
160 \\
310 \\
410 \\
480\end{array}$ & $\begin{array}{l}100 \\
260 \\
400 \\
460 \\
638\end{array}$ & $\begin{array}{l}\text { QTge } \\
\text { QTge, QTm } \\
\text { QTm } \\
\text { QTm } \\
\text { QTm }\end{array}$ & $\begin{array}{l}\text { silt and gravel; sandy silt; sand } \\
\text { gravel; sand and gravel; sand } \\
\text { sand; sandy silt and gravel } \\
\text { sand; sandy silt; sand and gravel } \\
\text { sand; sand and gravel; sandy silt }\end{array}$ \\
\hline 36 & 6/8-7D1 & 122.79500 & 38.38929 & 410 & 70 & -- & -- & QTm & -- \\
\hline 37 & $6 / 8-7 \mathrm{~K} 1$ & 122.78599 & 38.38124 & 340 & 70 & 0 & 260 & Qyal, QTm & sand and gravel fill; gravel; sandstone \\
\hline 38 & 6/8-7P1 & 122.78874 & 38.37788 & 346 & 95 & 305 & 41 & QTm & sandy clay; sand \\
\hline 39 & 6/8-8A1 & 122.76344 & 38.38781 & 248 & 90 & 30 & 218 & QTge, QTm & sandy clay; sand; sandy clay and gravel; clay and gravel \\
\hline 40 & $6 / 8-8 \mathrm{Q} 1$ & 122.76679 & 38.37784 & $200+$ & 75 & -- & -- & QTge, QTm(?) & -- \\
\hline 41 & $6 / 8-12 J 1$ & 122.68866 & 38.38138 & 223 & 130 & -- & -- & QTge & -- \\
\hline 42 & 6/8-12K1 & 122.69044 & 38.38110 & 205 & 120 & $\begin{array}{c}40 \\
100 \\
185\end{array}$ & $\begin{array}{l}20 \\
20 \\
20\end{array}$ & $\begin{array}{l}\text { QTge } \\
\text { QTge } \\
\text { QTge }\end{array}$ & $\begin{array}{l}\text { perforated interval } \\
\text { perforated interval } \\
\text { perforated interval }\end{array}$ \\
\hline 43 & 6/8-13R1 & 122.68656 & 38.36298 & 675 & 115 & -- & -- & Qyal, QTge, QTm(?) & - \\
\hline 44 & $6 / 8-15 \mathrm{~F} 1$ & 122.73556 & 38.36803 & 275 & 95 & $\begin{array}{c}50 \\
680 \\
\end{array}$ & $\begin{array}{c}600 \\
45 \\
\end{array}$ & $\begin{array}{l}\text { QTge, QTm } \\
\text { QTm }\end{array}$ & $\begin{array}{l}\text { silt and gravel; sandy silt; gravel; sand } \\
\text { gravel; muddy clay and gravel }\end{array}$ \\
\hline
\end{tabular}


Locations and data for water wells of the Santa Rosa Valley, Sonoma County, California

Data for selected water wells of the Santa Rosa Valley. Sonoma County, California-Continued

[--, no data; *, data for deepened well]

\begin{tabular}{|c|c|c|c|c|c|c|c|c|c|}
\hline $\begin{array}{c}\text { Map } \\
\text { Number }\end{array}$ & \begin{tabular}{|c|} 
Well \\
Number
\end{tabular} & \begin{tabular}{|c|} 
West \\
Longitude $\left({ }^{\circ}\right)$ \\
\end{tabular} & $\begin{array}{c}\text { North } \\
\text { Latitude }\left({ }^{\circ}\right) \\
\end{array}$ & $\begin{array}{c}\text { Total Depth } \\
\text { (feet) }\end{array}$ & \begin{tabular}{|c|} 
Surface \\
Elevation (feet) \\
\end{tabular} & $\begin{array}{c}\text { Depth to top of } \\
\text { water interval (feet) }\end{array}$ & $\begin{array}{l}\text { Water interval } \\
\text { thickness (feet) }\end{array}$ & \begin{tabular}{|c|} 
Geologic unit present \\
in water interval
\end{tabular} & Down-Hole Lithology of water interval \\
\hline 45 & 6/8-15Q1 & 122.73055 & 38.36343 & 914 & 95 & $\begin{array}{c}60 \\
400\end{array}$ & $\begin{array}{l}190 \\
300\end{array}$ & $\begin{array}{l}\text { QTge } \\
\text { QTge }\end{array}$ & $\begin{array}{l}\text { sand and gravel; silt and sand } \\
\text { silt, gravel, and redwood }\end{array}$ \\
\hline 46 & 6/8-15R1 & 122.72275 & 38.36382 & 1028 & 95 & $\begin{array}{c}20 \\
350 \\
380\end{array}$ & $\begin{array}{c}310 \\
10 \\
440\end{array}$ & $\begin{array}{l}\text { QTge } \\
\text { QTge } \\
\text { QTge }\end{array}$ & $\begin{array}{l}\text { silt and gravel; gravel and sand; silt and sand } \\
\text { medium gravel } \\
\text { gravel; sandy clay; sandy clay and gravel; silt and gravel }\end{array}$ \\
\hline 47 & 6/8-16F1 & 122.75346 & 38.37123 & 542 & 85 & -- & -- & -- & -- \\
\hline 48 & 6/8-16K1 & 122.75018 & 38.36531 & $\sim 710$ & 85 & -- & -- & QTge, QTm & -- \\
\hline 49 & 6/8-16R1 & 122.74245 & 38.36325 & 568 & 90 & -- & -- & QTge, QTm & -- \\
\hline 49 & 6/8-16R1* & 122.74245 & 38.36325 & 1204 & 90 & 749 & 11 & QTm & gravel and sand \\
\hline & & & & & & $\begin{array}{c}930 \\
1050 \\
1150\end{array}$ & $\begin{array}{c}7 \\
15 \\
54\end{array}$ & $\begin{array}{l}\text { QTm } \\
\text { QTm } \\
\text { QTm }\end{array}$ & $\begin{array}{l}\text { gravel and sand } \\
\text { sandy cemented gravel } \\
\text { clay and gravel; boulders }\end{array}$ \\
\hline 50 & 6/8-17K1 & 122.76827 & 38.36535 & 210 & 140 & -- & -- & QTm & -- \\
\hline 51 & 6/8-20G2 & 122.76466 & 38.35431 & 239 & 120 & -- & -- & QTm & -- \\
\hline 52 & $6 / 8-20 J 1$ & 122.76185 & 38.35131 & 302 & 125 & $\begin{array}{c}62 \\
104 \\
178\end{array}$ & $\begin{array}{l}24 \\
20 \\
12\end{array}$ & $\begin{array}{l}\text { QTm } \\
\text { QTm } \\
\text { QTm }\end{array}$ & $\begin{array}{l}\text { sandstone; sand and fine gravel } \\
\text { sand and gravel; sandstone } \\
\text { sand }\end{array}$ \\
\hline 53 & 6/8-20Q1 & 122.76785 & 38.34801 & 316 & 135 & -- & -- & QTm & -- \\
\hline 54 & $6 / 8-22 \mathrm{~N} 1$ & 122.73787 & 38.34765 & 200 & 120 & 185 & 15 & QTm & sandy clay; gravel \\
\hline 55 & $6 / 8-23 \mathrm{C} 1$ & 122.71337 & 38.35719 & 438 & 95 & -- & -- & QTge, QTm & -- \\
\hline 56 & $6 / 8-23 C 2$ & 122.71320 & 38.35701 & $300-350$ & 95 & -- & -- & QTge, QTm & perforated near 100 feet and below \\
\hline 57 & $6 / 8-26 \mathrm{~K} 1$ & 122.71263 & 38.33576 & 203 & 100 & -- & -- & QTm & -- \\
\hline 58 & $6 / 8-26 \mathrm{~N} 1$ & 122.71911 & 38.33414 & 246 & 105 & -- & -- & QTm & -- \\
\hline 59 & $6 / 8-26 \mathrm{~N} 2$ & 122.72160 & 38.33261 & 290 & 150 & -- & -- & QTm & -- \\
\hline 60 & 6/8-27P1 & 122.73537 & 38.33469 & 582 & 120 & $\begin{array}{l}360 \\
435 \\
498\end{array}$ & $\begin{array}{l}62 \\
38 \\
74\end{array}$ & $\begin{array}{l}\text { QTm } \\
\text { QTm } \\
\text { QTm }\end{array}$ & $\begin{array}{l}\text { sand; gravel } \\
\text { clay and gravel; hard cement gravel } \\
\text { gravel and streaks of clay; gravel }\end{array}$ \\
\hline 61 & 6/8-28E1 & 122.75813 & 38.33930 & 200 & 195 & 65 & 83 & QTm & sandstone; clam shells \\
\hline 62 & 6/8-29A1 & 122.75964 & 38.34580 & 259 & 135 & -- & -- & QTm(?) & upper perforations above 104 feet \\
\hline 63 & 6/8-29B1 & 122.76646 & 38.34332 & 1949 & 140 & -- & -- & QTm(?) & -- \\
\hline 64 & 6/8-29E1 & 122.77621 & 38.34246 & 285 & 200 & -- & -- & QTm(?) & -- \\
\hline 65 & 6/8-30F1 & 122.78764 & 38.34069 & 248 & 290 & 12 & 234 & QTm & sand \\
\hline 66 & $6 / 8-30 F 2$ & 122.79130 & 38.34196 & 288 & 290 & $\begin{array}{c}95 \\
219\end{array}$ & $\begin{array}{l}58 \\
69\end{array}$ & $\begin{array}{l}\text { QTm } \\
\text { QTm }\end{array}$ & $\begin{array}{l}\text { sand } \\
\text { sandstone; sand and gravel }\end{array}$ \\
\hline 67 & 6/8-32B1 & 122.76675 & 38.33124 & 202 & 310 & -- & -- & Tsv & -- \\
\hline 68 & 6/8-33E1 & 122.75414 & 38.32449 & 215 & 250 & -- & -- & $\mathrm{Tp}$ & -- \\
\hline 69 & 6/8-33M1 & 122.75441 & 38.32236 & 261 & 210 & 100 & 28 & QTm & clay and gravel, mixed \\
\hline
\end{tabular}


Locations and data for water wells of the Santa Rosa Valley, Sonoma County, California

Table 1. Data for selected water wells of the Santa Rosa Valley, Sonoma County, California-Continued

[--, no data; *, data for deepened well]

\begin{tabular}{|c|c|c|c|c|c|c|c|c|c|}
\hline $\begin{array}{c}\text { Map } \\
\text { Number }\end{array}$ & $\begin{array}{c}\text { Well } \\
\text { Number }\end{array}$ & $\begin{array}{c}\text { West } \\
\text { Longitude }\left({ }^{\circ}\right)\end{array}$ & $\begin{array}{c}\text { North } \\
\text { Latitude }\left({ }^{\circ}\right)\end{array}$ & $\begin{array}{l}\text { Total Depth } \\
\text { (feet) }\end{array}$ & \begin{tabular}{|c|} 
Surface \\
Elevation (feet)
\end{tabular} & $\begin{array}{c}\text { Depth to top of } \\
\text { water interval (feet) }\end{array}$ & $\begin{array}{l}\text { Water interval } \\
\text { thickness (feet) }\end{array}$ & \begin{tabular}{|c|}
$\begin{array}{c}\text { Geologic unit present } \\
\text { in water interval }\end{array}$ \\
\end{tabular} & Down-Hole Lithology of water interval \\
\hline 70 & 6/8-33P1 & 122.74917 & 38.31906 & 338 & 265 & 118 & 27 & QTm & sand; sand and fine gravel \\
\hline 71 & 6/8-34G1 & 122.72627 & 38.32597 & 213 & 190 & 207 & 6 & $\operatorname{Tsv}(?)$ & rock \\
\hline 72 & $6 / 8-35 \mathrm{~A} 2$ & 122.70557 & 38.33031 & 660 & 110 & -- & -- & QTm & -- \\
\hline 73 & $6 / 8-35 \mathrm{~F} 1$ & 122.71399 & 38.32486 & 509 & 165 & $\begin{array}{c}76 \\
139 \\
153 \\
308 \\
409 \\
426\end{array}$ & $\begin{array}{c}20 \\
4 \\
7 \\
54 \\
15 \\
21\end{array}$ & $\begin{array}{l}\text { QTm } \\
\text { QTm } \\
\text { QTm } \\
\text { QTm } \\
\text { QTm } \\
\text { QTm }\end{array}$ & $\begin{array}{l}\text { gravel } \\
\text { sand and gravel } \\
\text { clay and gravel; cemented gravel } \\
\text { gravel; clay and gravel } \\
\text { gravel } \\
\text { gravel }\end{array}$ \\
\hline 74 & 6/8-35L1 & 122.71489 & 38.32246 & 288 & 155 & -- & -- & QTm & -- \\
\hline 75 & 6/8-36A1 & 122.68749 & 38.32797 & 695 & 115 & $\begin{array}{c}60 \\
178 \\
324 \\
376 \\
471 \\
506\end{array}$ & $\begin{array}{c}4 \\
7 \\
14 \\
48 \\
35 \\
6\end{array}$ & $\begin{array}{l}\text { QTm(?) } \\
\text { QTm } \\
\text { QTm } \\
\text { QTm } \\
\text { QTm } \\
\text { QTm }\end{array}$ & $\begin{array}{l}\text { clay and gravel } \\
\text { clay and little fine gravel } \\
\text { cemented gravel } \\
\text { cemented gravel; sandy clay } \\
\text { sandy clay; gravel } \\
\text { cemented gravel }\end{array}$ \\
\hline 76 & 6/8-36P1 & 122.69433 & 38.31866 & 255 & 130 & 237 & 18 & QTm & sand and gravel; gravel \\
\hline 77 & 6/9-1P2 & 122.80789 & 38.39163 & 248 & 90 & 25 & 223 & QTm & "quicksand"; fine gravel \\
\hline 78 & $6 / 9-2 \mathrm{C} 1$ & 122.82629 & 38.40111 & 600 & 120 & $\begin{array}{l}304 \\
429\end{array}$ & $\begin{array}{c}95 \\
171\end{array}$ & $\begin{array}{l}\text { QTm } \\
\text { QTm }\end{array}$ & $\begin{array}{l}\text { clam shells and sand } \\
\text { shells and sand; sand, grit, and shells; sediment and sand }\end{array}$ \\
\hline 79 & $6 / 9-2 \mathrm{~F} 1$ & 122.82613 & 38.40006 & 260 & 125 & 2 & 260 & QTm & soft quicksand; dry sand; clam shells \\
\hline 80 & $6 / 9-2 \mathrm{P} 1$ & 122.82460 & 38.39350 & 215 & 200 & 200 & 15 & QTm & sand \\
\hline 81 & 6/9-3A1 & 122.83726 & 38.40224 & 260 & 230 & 250 & 10 & QTm & sandstone \\
\hline 82 & 6/9-3A2 & 122.83508 & 38.40189 & 305 & 210 & 293 & 12 & QTm & black sand \\
\hline 83 & 6/9-3H1 & 122.83589 & 38.40034 & 295 & 240 & 4 & 291 & QTm & sandstone; sand; gravel \\
\hline 84 & 6/9-4M1 & 122.86605 & 38.39546 & 230 & 365 & 40 & 100 & QTm & sandstone \\
\hline 85 & 6/9-7F1 & 122.90025 & 38.38701 & 280 & 725 & 3 & 277 & QTm & sandstone \\
\hline 86 & 6/9-9E1 & 122.86870 & 38.38382 & 280 & 435 & 0 & 280 & QTm & sandstone; sand \\
\hline 87 & 6/9-9N1 & 122.86690 & 38.37944 & 250 & 520 & 16 & 234 & QTm & sandstone \\
\hline 88 & 6/9-10H1 & 122.83424 & 38.38580 & 400 & 315 & 178 & 222 & QTm & clay and sandstone; "sticky stone"; sandstone \\
\hline 89 & 6/9-11A3 & 122.81870 & 38.38626 & 220 & 170 & 105 & 115 & QTm & sand; sandstone \\
\hline 90 & 6/9-11B1 & 122.82190 & 38.39013 & 420 & 170 & 50 & 370 & QTm & fine sand; fine sand and gravel \\
\hline 91 & 6/9-11C1 & 122.82531 & 38.38921 & 220 & 225 & 155 & 65 & QTm & sandstone; sand and gravel \\
\hline 92 & 6/9-11D1 & 122.82884 & 38.38638 & 280 & 340 & 175 & 105 & QTm & sandstone \\
\hline 93 & 6/9-11D2 & 122.83041 & 38.39031 & 338 & 225 & 278 & 60 & QTm & sandstone \\
\hline 94 & 6/9-11F1 & 122.82607 & 38.38581 & 235 & 330 & 142 & 93 & QTm & sandstone \\
\hline 95 & $6 / 9-12 \mathrm{D} 3$ & 122.81446 & 38.38892 & 278 & 130 & 35 & 243 & QTm & "quicksand" and clamshells; "quicksand"; partly-cemented clamshells \\
\hline
\end{tabular}


Locations and data for water wells of the Santa Rosa Valley, Sonoma County, California

by Zenon C. Valin and Robert J. McLaughlin

Table 1. Data for selected water wells of the Santa Rosa Vallev, Sonoma County, California-Continued

[--, no data; *, data for deepened well]

\begin{tabular}{|c|c|c|c|c|c|c|c|c|c|}
\hline $\begin{array}{c}\text { Map } \\
\text { Number }\end{array}$ & \begin{tabular}{|c|} 
Well \\
Number \\
\end{tabular} & $\begin{array}{c}\text { West } \\
\text { Longitude }\left({ }^{\circ}\right) \\
\end{array}$ & $\begin{array}{c}\text { North } \\
\text { Latitude }\left({ }^{\circ}\right) \\
\end{array}$ & $\begin{array}{c}\text { Total Depth } \\
\text { (feet) }\end{array}$ & \begin{tabular}{|c|} 
Surface \\
Elevation (feet)
\end{tabular} & $\begin{array}{c}\text { Depth to top of } \\
\text { water interval (feet) }\end{array}$ & $\begin{array}{l}\text { Water interval } \\
\text { thickness (feet) }\end{array}$ & $\mid \begin{array}{c}\text { Geologic unit present } \\
\text { in water interval }^{1}\end{array}$ & Down-Hole Lithology of water interval \\
\hline 95 & $6 / 9-12 \mathrm{D} 3$ & 122.81446 & 38.38892 & 278 & 130 & 35 & 243 & QTm & "quicksand" and clamshells; "quicksand"; partly-cemented clamshells \\
\hline 96 & 6/9-13B1 & 122.80280 & 38.37484 & 212 & 175 & 200 & 12 & QTm & sand; gravel and sand \\
\hline 97 & $6 / 9-13 \mathrm{C} 1$ & 122.80820 & 38.37476 & 204 & 235 & 32 & 172 & QTm & "quicksand"; gravel \\
\hline 98 & 6/9-13D1 & 122.81100 & 38.37305 & 471 & 260 & -- & -- & QTm & -- \\
\hline 99 & $6 / 9-13 \mathrm{~J} 1$ & 122.79914 & 38.36738 & 280 & 200 & 239 & 41 & QTm & sand and gravel \\
\hline 100 & 6/9-13L1 & 122.80865 & 38.36625 & 360 & 260 & 0 & 360 & QTm & "quicksand"; sand and gravel; sand \\
\hline 101 & $6 / 9-14 \mathrm{C} 1$ & 122.82755 & 38.37205 & 365 & 325 & -- & -- & QTm & -- \\
\hline 102 & 6/9-14J1 & 122.83302 & 38.36760 & 394 & 330 & -- & -- & QTm & -- \\
\hline 103 & 6/9-14R2 & 122.81895 & 38.36362 & 200 & 325 & -- & -- & QTm & -- \\
\hline 104 & $6 / 9-15 \mathrm{D} 1$ & 122.84695 & 38.37424 & 200 & 395 & $\begin{array}{c}25 \\
116\end{array}$ & $\begin{array}{l}67 \\
84\end{array}$ & $\begin{array}{l}\text { QTm } \\
\text { QTm }\end{array}$ & $\begin{array}{l}\text { sandstone; sand } \\
\text { clay and sandstone; sandstone and clamshells }\end{array}$ \\
\hline 105 & 6/9-15G1 & 122.84074 & 38.36898 & 200 & 440 & 65 & 135 & QTm & sandstone \\
\hline 106 & 6/9-22A1 & 122.83392 & 38.35924 & 360 & 120 & 20 & 340 & QTm & sandstone; sandstone containing shells; sand and shells \\
\hline 107 & 7/6-19F1 & 122.56850 & 38.44087 & 585 & 575 & 35 & 530 & QTge, Tsv(?) & clay and gravel \\
\hline 108 & $7 / 6-19 \mathrm{~N} 2$ & 122.57348 & 38.43269 & 334 & 475 & -- & -- & QTge & -- \\
\hline 109 & 7/6-19R1 & 122.55943 & 38.43305 & 300 & 465 & $\begin{array}{l}220 \\
260\end{array}$ & $\begin{array}{l}40 \\
40\end{array}$ & $\begin{array}{l}\text { QTge } \\
\text { Tsv(?) }\end{array}$ & $\begin{array}{l}\text { small boulders and coarse sand } \\
\text { volcanic ash }\end{array}$ \\
\hline 110 & 7/6-29M1 & 122.54590 & 38.42226 & 400 & 415 & -- & -- & Qyal, QTge(?), Tsv(?) & -- \\
\hline 111 & 7/6-31P1 & 122.56981 & 38.40244 & 205 & 470 & -- & -- & Tsv & -- \\
\hline 112 & 7/6-32A2 & 122.53902 & 38.41571 & 390 & 445 & -- & -- & Tsv & -- \\
\hline 113 & $7 / 6-32 \mathrm{H} 1$ & 122.54095 & 38.41085 & 406 & 415 & 100 & 300 & Tsv & perforated interval \\
\hline 114 & $7 / 7-5 \mathrm{~F} 2$ & 122.66081 & 38.48391 & 275 & 325 & $\begin{array}{c}65 \\
160\end{array}$ & $\begin{array}{l}10 \\
15\end{array}$ & $\begin{array}{l}\text { QTge } \\
\text { QTge }\end{array}$ & $\begin{array}{l}\text { packed gravel } \\
\text { medium packed gravel }\end{array}$ \\
\hline 115 & 7/7-6G1 & 122.67445 & 38.48309 & 338 & 295 & $\begin{array}{c}3 \\
87 \\
124 \\
172 \\
310\end{array}$ & $\begin{array}{l}38 \\
21 \\
17 \\
31 \\
23\end{array}$ & $\begin{array}{l}\text { QTge } \\
\text { QTge } \\
\text { QTge } \\
\text { QTge } \\
\text { QTge }\end{array}$ & $\begin{array}{l}\text { fine sand; clay streaked by gravel } \\
\text { sand and gravel } \\
\text { sand and gravel } \\
\text { clay streaked by gravel } \\
\text { sand and gravel }\end{array}$ \\
\hline 116 & 7/7-7F2 & 122.68053 & 38.46873 & 320 & 245 & -- & -- & Tsv & -- \\
\hline 117 & 7/7-7L1 & 122.67903 & 38.46777 & 214 & 240 & -- & -- & Tsv & -- \\
\hline 118 & 7/7-7N1 & 122.68272 & 38.46280 & 204 & 250 & -- & -- & Tsv & -- \\
\hline 119 & 7/7-7R3 & 122.66928 & 38.46354 & 245 & 260 & 228 & 17 & Tsv & perforated interval \\
\hline 120 & 7/7-8A1 & 122.65257 & 38.47384 & 385 & 315 & -- & -- & QTge & -- \\
\hline 121 & 7/7-8A2 & 122.64922 & 38.47240 & 230 & 360 & $\begin{array}{l}210 \\
224\end{array}$ & $\begin{array}{l}7 \\
4\end{array}$ & $\begin{array}{l}\text { QTge } \\
\text { QTge }\end{array}$ & $\begin{array}{l}\text { cemented gravel; gravel } \\
\text { clay and gravel }\end{array}$ \\
\hline 122 & $7 / 7-8 \mathrm{G} 2$ & 122.65400 & 38.47124 & 425 & 290 & -- & -- & QTge & -- \\
\hline 123 & 7/7-8L1 & 122.66128 & 38.46521 & 278 & 250 & 208 & 15 & Tsv & volcanic ash \\
\hline
\end{tabular}


Locations and data for water wells of the Santa Rosa Valley, Sonoma County, California

by Zenon C. Valin and Robert J. McLaughlin

Table 1. Data for selected water wells of the Santa Rosa Valley, Sonoma County, California-Continued

[--, no data; *, data for deepened well]

\begin{tabular}{|c|c|c|c|c|c|c|c|c|c|}
\hline $\begin{array}{c}\text { Map } \\
\text { Number }\end{array}$ & $\begin{array}{c}\text { Well } \\
\text { Number }\end{array}$ & $\begin{array}{c}\text { West } \\
\text { Longitude }\left({ }^{\circ}\right) \\
\end{array}$ & $\begin{array}{c}\text { North } \\
\text { Latitude }\left({ }^{\circ}\right) \\
\end{array}$ & $\begin{array}{c}\text { Total Depth } \\
\text { (feet) }\end{array}$ & \begin{tabular}{|c|}
$\begin{array}{c}\text { Surface } \\
\text { Elevation (feet) }\end{array}$ \\
\end{tabular} & $\begin{array}{c}\text { Depth to top of } \\
\text { water interval (feet) }\end{array}$ & $\begin{array}{l}\text { Water interval } \\
\text { thickness (feet) }\end{array}$ & $\begin{array}{c}\text { Geologic unit present } \\
\text { in water interval }\end{array}$ & Down-Hole Lithology of water interval \\
\hline 124 & 7/7-8M2 & 122.66658 & 38.46786 & 211 & 250 & -- & -- & -- & -- \\
\hline 125 & 7/7-8P2 & 122.66132 & 38.46433 & 250 & 285 & 233 & 17 & Tsv & volcanic ash \\
\hline 126 & 7/7-9D1 & 122.64804 & 38.47239 & 245 & 380 & $\begin{array}{c}35 \\
230\end{array}$ & $\begin{array}{l}10 \\
15\end{array}$ & $\begin{array}{l}\text { QTge } \\
\text { QTge }\end{array}$ & $\begin{array}{l}\text { fine packed gravel and sand } \\
\text { coarse gravelly sand }\end{array}$ \\
\hline 127 & 7/7-9D2 & 122.64752 & 38.47370 & 323 & 375 & 275 & 25 & QTge & hard gravel; loose gravel \\
\hline 128 & 7/7-9D3 & 122.64786 & 38.47306 & 319 & 400 & $\begin{array}{l}70 \\
241 \\
252 \\
271 \\
289\end{array}$ & $\begin{array}{c}60 \\
2 \\
4 \\
4 \\
20\end{array}$ & $\begin{array}{l}\text { QTge } \\
\text { QTge } \\
\text { QTge } \\
\text { QTge } \\
\text { QTge }\end{array}$ & $\begin{array}{l}\text { sandy clay and gravel; cemented gravel } \\
\text { gravel } \\
\text { cemented gravel } \\
\text { sandy clay, some gravel } \\
\text { cemented gravel }\end{array}$ \\
\hline 129 & 7/7-10M1 & 122.62962 & 38.46587 & $\sim 300$ & 525 & -- & -- & QTge & -- \\
\hline 130 & 7/7-13N1 & 122.59015 & 38.44518 & 490 & 475 & -- & -- & $\operatorname{Tsv}(?)$ & -- \\
\hline 131 & 7/7-14P1 & 122.60632 & 38.44630 & 828 & 430 & -- & -- & Qyal, QTge & -- \\
\hline 132 & $7 / 7-15 \mathrm{C} 1$ & 122.62458 & 38.46004 & 397 & 375 & -- & -- & QTge & -- \\
\hline 133 & $7 / 7-15 \mathrm{C} 2$ & 122.62312 & 38.45704 & 205 & 365 & $\begin{array}{c}40 \\
150 \\
185\end{array}$ & $\begin{array}{l}25 \\
20 \\
20\end{array}$ & $\begin{array}{l}\text { QTge } \\
\text { QTge } \\
\text { QTge }\end{array}$ & $\begin{array}{l}\text { sand, gravel, and clay; pea gravel } \\
\text { sand and gravel } \\
\text { sand and gravel }\end{array}$ \\
\hline 134 & 7/7-15M1 & 122.62655 & 38.45215 & 250 & 435 & -- & -- & QTge & -- \\
\hline 135 & 7/7-16A1 & 122.63469 & 38.46018 & 263 & 325 & 50 & 263 & QTge & perforated interval \\
\hline 136 & $7 / 7-16 C 2$ & 122.63984 & 38.45958 & 278 & 310 & -- & -- & QTge(?) & all casing perforated \\
\hline 137 & 7/7-17A1 & 122.65269 & 38.46022 & 846 & 290 & -- & -- & Tsv, QTm(?) & -- \\
\hline 138 & 7/7-17A2 & 122.65000 & 38.45908 & 1018 & 285 & -- & -- & Tsv, QTm(?) & -- \\
\hline 139 & 7/7-18J1 & 122.66829 & 38.44996 & $300+$ & 215 & -- & -- & -- & -- \\
\hline 140 & 7/7-18Q1 & 122.67381 & 38.44691 & 249 & 205 & -- & -- & Qyal, Qoal(?) & all casing perforated \\
\hline 141 & 7/7-18R2 & 122.66988 & 38.44674 & 206 & 210 & -- & -- & Qoal(?), Tsv & below $50 \mathrm{ft}$. alternate casing joints are perforated \\
\hline 142 & 7/7-19Q1 & 122.67288 & 38.43333 & 680 & 225 & -- & -- & Qyal, Tsv & -- \\
\hline 143 & 7/7-19R1 & 122.66997 & 38.43301 & 670 & 225 & -- & -- & Qyal, Tsv & -- \\
\hline 144 & 7/7-20P1 & 122.66024 & 38.43401 & 243 & 245 & -- & -- & Tsv & uncased below $40 \mathrm{ft}$. \\
\hline 145 & 7/7-23A1 & 122.59335 & 38.44377 & 365 & 475 & $\begin{array}{l}250 \\
303\end{array}$ & $\begin{array}{l}50 \\
62\end{array}$ & $\begin{array}{l}\text { QTge } \\
\text { QTge }\end{array}$ & $\begin{array}{l}\text { clay and gravel; gravel; sandy clay and gravel } \\
\text { sand and gravel; cemented gravel; sandy clay and gravel }\end{array}$ \\
\hline 146 & 7/7-23A2 & 122.59446 & 38.44262 & 337 & 450 & -- & -- & QTge & -- \\
\hline 147 & $7 / 7-23 \mathrm{C} 1$ & 122.60417 & 38.44402 & $1300+$ & 420 & -- & -- & Qoal(?), QTge & -- \\
\hline 148 & 7/7-23F1 & 122.60683 & 38.44159 & 440 & 420 & -- & -- & Qoal(?), QTge & below $75 \mathrm{ft}$. alternate $20 \mathrm{ft}$. joints are perforated \\
\hline 149 & 7/7-23G1 & 122.59795 & 38.44168 & $1100+$ & 430 & -- & -- & QTge & -- \\
\hline 150 & 7/7-24A1 & 122.57794 & 38.44297 & 622 & 565 & -- & -- & Tsv & cased to $208 \mathrm{ft}$.; no perforations \\
\hline 151 & 7/7-24B1 & 122.57932 & 38.44366 & 585 & 565 & -- & -- & -- & -- \\
\hline 152 & 7/7-25A1 & 122.57757 & 38.42849 & $400+$ & 450 & -- & -- & -- & -- \\
\hline
\end{tabular}


Locations and data for water wells of the Santa Rosa Valley, Sonoma County, California

Table 1. Data for selected water wells of the Santa Rosa Valley, Sonoma County, California-Continued

[--, no data; *, data for deepened well]

\begin{tabular}{|c|c|c|c|c|c|c|c|c|c|}
\hline $\begin{array}{l}\text { Map } \\
\text { Number }\end{array}$ & $\begin{array}{c}\text { Well } \\
\text { Number }\end{array}$ & \begin{tabular}{|c|} 
West \\
Longitude $\left({ }^{\circ}\right)$
\end{tabular} & $\begin{array}{c}\text { North } \\
\text { Latitude }\left({ }^{\circ}\right)\end{array}$ & $\begin{array}{l}\text { Total Depth } \\
\text { (feet) }\end{array}$ & $\begin{array}{c}\text { Surface } \\
\text { Elevation (feet) }\end{array}$ & $\begin{array}{c}\text { Depth to top of } \\
\text { water interval (feet) }\end{array}$ & $\begin{array}{l}\text { Water interval } \\
\text { thickness (feet) }\end{array}$ & \begin{tabular}{|c|} 
Geologic unit present \\
in water interval
\end{tabular} & Down-Hole Lithology of water interval \\
\hline 153 & $7 / 7-29 \mathrm{~B} 1$ & 122.65459 & 38.43046 & 240 & 380 & -- & -- & Tsv & -- \\
\hline 154 & 7/7-29D1 & 122.66563 & 38.43071 & 588 & 240 & $\begin{array}{c}40 \\
130 \\
410\end{array}$ & $\begin{array}{c}20 \\
50 \\
130\end{array}$ & $\begin{array}{l}\text { Qoal(?) } \\
\text { QTge(?) } \\
\text { Tsv }\end{array}$ & $\begin{array}{l}\text { loose gravel } \\
\text { sand and gravel; shale; boulders } \\
\text { volcanic ash }\end{array}$ \\
\hline 155 & 7/7-29L1 & 122.65847 & 38.42253 & 365 & 265 & -- & -- & Tsv & -- \\
\hline 156 & 7/7-29L2 & 122.65847 & 38.42253 & 365 & 265 & -- & -- & Tsv & -- \\
\hline 157 & 7/7-30C1 & 122.67705 & 38.42917 & 448 & 255 & -- & -- & QTge, QTm(?) & -- \\
\hline 158 & 7/7-32B1 & 122.65569 & 38.41571 & 266 & 325 & -- & -- & Tsv & -- \\
\hline 159 & $7 / 7-32 \mathrm{G} 1$ & 122.65392 & 38.41109 & 403 & 325 & $\begin{array}{c}0 \\
210\end{array}$ & $\begin{array}{c}40 \\
182\end{array}$ & $\begin{array}{l}\text { Qyal } \\
\text { Tsv }\end{array}$ & $\begin{array}{l}\text { gravel } \\
\text { volcanic ash; volcanic ash and basalt; ash and clay }\end{array}$ \\
\hline 160 & 7/7-33R1 & 122.63113 & 38.40386 & 438 & 510 & 82 & 95 & Tsv(?) & cemented gravel \\
\hline 161 & 7/7-36A1 & 122.57607 & 38.41352 & 284 & 615 & $\begin{array}{c}60 \\
177\end{array}$ & $\begin{array}{c}45 \\
9\end{array}$ & $\begin{array}{l}\operatorname{Tsv}(?) \\
\operatorname{Tsv}(?)\end{array}$ & $\begin{array}{l}\text { gravel; coarse gravel; clay and gravel } \\
\text { boulders }\end{array}$ \\
\hline 162 & $7 / 8-3 \mathrm{C} 1$ & 122.73398 & 38.48720 & 235 & 175 & 47 & 68 & OTge & clay and gravel \\
\hline 163 & $7 / 8-6 \mathrm{H} 2$ & 122.78001 & 38.48443 & 397 & 135 & $\begin{array}{l}26 \\
145 \\
232 \\
275\end{array}$ & $\begin{array}{c}56 \\
42 \\
3 \\
55\end{array}$ & $\begin{array}{l}\text { QTge } \\
\text { QTge } \\
\text { QTge } \\
\text { QTge }\end{array}$ & $\begin{array}{l}\text { sandy clay; sandstone; loose boulders } \\
\text { gravelly clay } \\
\text { small loose boulders } \\
\text { hard sandstone; gravelly clay; loose sandstone }\end{array}$ \\
\hline 164 & 7/8-9D1 & 122.75932 & 38.47237 & 200 & 130 & -- & -- & QTge & -- \\
\hline 165 & 7/8-9R1 & 122.74506 & 38.46287 & 210 & 120 & $\begin{array}{c}35 \\
136 \\
162 \\
180\end{array}$ & $\begin{array}{l}15 \\
3 \\
3 \\
7\end{array}$ & $\begin{array}{l}\text { QTge } \\
\text { QTge } \\
\text { QTge } \\
\text { QTge }\end{array}$ & $\begin{array}{l}\text { sand and gravel } \\
\text { gravel } \\
\text { gravel } \\
\text { gravel }\end{array}$ \\
\hline 166 & 7/8-10L1 & 122.73494 & 38.46586 & 212 & 135 & 100 & 14 & QTge & light gravel and some coarse sand \\
\hline 167 & 7/8-11G1 & 122.71252 & 38.47143 & 237 & 290 & -- & -- & QTge & gravel \\
\hline 168 & 7/8-11K1 & 122.71213 & 38.46664 & 254 & 300 & 238 & 16 & QTge & sandstone \\
\hline 169 & 7/8-12E2 & 122.70345 & 38.46958 & 298 & 245 & 80 & 218 & Tsv & perforated interval \\
\hline 170 & $7 / 8-12 \mathrm{H} 1$ & 122.68862 & 38.46972 & 255 & 460 & $\begin{array}{l}160 \\
208\end{array}$ & $\begin{array}{l}24 \\
47\end{array}$ & $\begin{array}{l}\text { Tsv } \\
\text { Tsv }\end{array}$ & $\begin{array}{l}\text { perforated interval } \\
\text { perforated interval }\end{array}$ \\
\hline 171 & 7/8-12K1 & 122.69168 & 38.46543 & 347 & 480 & 273 & 74 & Tsv & sand, clay, and gravel; sand and rock \\
\hline 172 & $7 / 8-12 \mathrm{Q} 1$ & 122.69530 & 38.46357 & 200 & 405 & -- & -- & Tsv & -- \\
\hline 173 & $7 / 8-12 \mathrm{Q} 2$ & 122.69440 & 38.46484 & 220 & 430 & 125 & 95 & Tsv & sandy clay and gravel; clay and gravel \\
\hline 174 & 7/8-13J1 & 122.68709 & 38.45319 & 253 & 20 & 148 & 105 & QTge(?) & perforated interval \\
\hline 175 & $7 / 8-13 J 2$ & 122.68858 & 38.45215 & 210 & 210 & 188 & 18 & $\operatorname{Tsv}(?)$ & perforated interval \\
\hline 176 & $7 / 8-13 \mathrm{~K} 1$ & 122.69300 & 38.45081 & 529 & 200 & $\begin{array}{l}129 \\
272\end{array}$ & $\begin{array}{c}6 \\
159\end{array}$ & $\begin{array}{l}\text { QTge } \\
\text { QTge }\end{array}$ & $\begin{array}{l}\text { cemented gravel } \\
\text { clay and gravel; cemented gravel; coarse gravel; clay }\end{array}$ \\
\hline 177 & 7/8-14A1 & 122.70570 & 38.46109 & 360 & 175 & -- & -- & QTge & -- \\
\hline
\end{tabular}


Locations and data for water wells of the Santa Rosa Valley, Sonoma County, California

Table 1. Data for selected water wells of the Santa Rosa Valley, Sonoma County, California-Continued

$[--$, no data; $*$, data for deepened well $]$

\begin{tabular}{|c|c|c|c|c|c|c|c|c|c|}
\hline $\begin{array}{l}\text { Map } \\
\text { Number }\end{array}$ & $\begin{array}{c}\text { Well } \\
\text { Number }\end{array}$ & $\begin{array}{c}\text { West } \\
\text { Longitude }\left(^{\circ}\right)\end{array}$ & $\begin{array}{c}\text { North } \\
\text { Latitude }\left({ }^{\circ}\right)\end{array}$ & $\begin{array}{l}\text { Total Depth } \\
\text { (feet) }\end{array}$ & \begin{tabular}{|c|} 
Surface \\
Elevation (feet)
\end{tabular} & $\begin{array}{c}\text { Depth to top of } \\
\text { water interval (feet) }\end{array}$ & $\begin{array}{l}\text { Water interval } \\
\text { thickness (feet) }\end{array}$ & $\begin{array}{c}\text { Geologic unit present } \\
\text { in water interval }\end{array}$ & Down-Hole Lithology of water interval \\
\hline 178 & 7/8-14A1 & 122.70570 & 38.46109 & 901 & 175 & -- & -- & Tsv & -- \\
\hline 179 & 7/8-14D1 & 122.72001 & 38.45855 & 300 & 155 & $\begin{array}{c}95 \\
146 \\
265\end{array}$ & $\begin{array}{l}15 \\
54 \\
35\end{array}$ & $\begin{array}{l}\text { QTge } \\
\text { QTge } \\
\text { QTge }\end{array}$ & $\begin{array}{l}\text { cemented gravel } \\
\text { gravel; clay and gravel } \\
\text { hard cemented gravel; hard clay and gravel }\end{array}$ \\
\hline 180 & 7/8-14E1 & 122.71919 & 38.45637 & 761 & 155 & -- & -- & QTge & -- \\
\hline 181 & 7/8-14M1 & 122.71815 & 38.45332 & 227.8 & 155 & -- & -- & QTge & -- \\
\hline 182 & 7/8-17N1 & 122.77569 & 38.44807 & 544 & 85 & $\begin{array}{c}3 \\
52 \\
162 \\
234 \\
376 \\
424 \\
506\end{array}$ & $\begin{array}{c}33 \\
10 \\
2 \\
34 \\
8 \\
6 \\
10\end{array}$ & $\begin{array}{l}\text { Qyal } \\
\text { QTge } \\
\text { QTge } \\
\text { QTge } \\
\text { QTge } \\
\text { QTge } \\
\text { QTge }\end{array}$ & $\begin{array}{l}\text { soft sandstone; gravel } \\
\text { gravel } \\
\text { cemented gravel } \\
\text { sandy clay; soft streaks with gravel; cemented gravel } \\
\text { cemented gravel } \\
\text { cemented gravel } \\
\text { sandstone }\end{array}$ \\
\hline 183 & 7/8-18K1 & 122.78579 & 38.45327 & 420 & 85 & $\begin{array}{c}20 \\
40 \\
330\end{array}$ & $\begin{array}{c}14 \\
2 \\
86\end{array}$ & $\begin{array}{l}\text { QTge(?) } \\
\text { QTge } \\
\text { QTge }\end{array}$ & $\begin{array}{l}\text { sand and gravel } \\
\text { gravel } \\
\text { gravel }\end{array}$ \\
\hline 184 & 7/8-18M1 & 122.79285 & 38.45500 & 640 & 80 & -- & -- & QTge & -- \\
\hline 185 & 7/8-18Q1 & 122.78283 & 38.45112 & 811 & 80 & $\begin{array}{c}50 \\
130 \\
192 \\
222\end{array}$ & $\begin{array}{c}5 \\
23 \\
6 \\
14\end{array}$ & $\begin{array}{l}\text { QTge } \\
\text { QTge } \\
\text { QTge } \\
\text { QTge }\end{array}$ & $\begin{array}{l}\text { sandy clay and gravel } \\
\text { cemented gravel; sand and clay } \\
\text { coarse cemented gravel } \\
\text { clay and gravel }\end{array}$ \\
\hline 186 & 7/8-19P1 & 122.78796 & 38.43610 & 245 & 85 & $\begin{array}{l}20 \\
45 \\
150 \\
219\end{array}$ & $\begin{array}{l}12 \\
20 \\
30 \\
16\end{array}$ & $\begin{array}{l}\text { QTge } \\
\text { QTge } \\
\text { QTm } \\
\text { QTge }\end{array}$ & $\begin{array}{l}\text { sand; gravel } \\
\text { gravel; cemented gravel } \\
\text { sand, gravel; sand, sediment } \\
\text { gravel }\end{array}$ \\
\hline 187 & 7/8-20C1 & 122.77245 & 38.44497 & 625 & 90 & $\begin{array}{c}77 \\
230 \\
539 \\
580 \\
591\end{array}$ & $\begin{array}{l}3 \\
1 \\
3 \\
2 \\
32\end{array}$ & $\begin{array}{l}\text { QTge } \\
\text { QTge } \\
\text { QTge } \\
\text { QTge } \\
\text { QTge }\end{array}$ & $\begin{array}{l}\text { gravel } \\
\text { gravel } \\
\text { gravel } \\
\text { gravel } \\
\text { sand containing trace of soft clay; gravel }\end{array}$ \\
\hline 188 & 7/8-20D1 & 122.77547 & 38.44410 & 484 & 90 & $\begin{array}{l}190 \\
446\end{array}$ & $\begin{array}{c}104 \\
30\end{array}$ & $\begin{array}{l}\text { QTge } \\
\text { QTge }\end{array}$ & $\begin{array}{l}\text { clay and gravel } \\
\text { cemented gravel }\end{array}$ \\
\hline 189 & 7/8-20K1 & 122.76485 & 38.43878 & 626 & 98 & -- & -- & QTge & -- \\
\hline 190 & 7/8-21C1 & 122.75147 & 38.44493 & 310 & 110 & -- & -- & QTge & coarse gravel \\
\hline 191 & $7 / 8-22 \mathrm{~K} 1$ & 122.73180 & 38.43643 & 285 & 135 & -- & -- & -- & -- \\
\hline 192 & 7/8-22Q1 & 122.72942 & 38.43433 & 360 & 140 & -- & -- & -- & -- \\
\hline 193 & $7 / 8-22 \mathrm{R} 2$ & 122.72568 & 38.43475 & 205 & 145 & -- & -- & -- & -- \\
\hline
\end{tabular}


Locations and data for water wells of the Santa Rosa Valley, Sonoma County, California

by Zenon C. Valin and Robert J. McLaughlin

Table 1. Data for selected water wells of the Santa Rosa Valley, Sonoma County, California-Continued

$[--$, no data; *, data for deepened well]

\begin{tabular}{|c|c|c|c|c|c|c|c|c|c|}
\hline $\begin{array}{l}\text { Map } \\
\text { Number }\end{array}$ & $\begin{array}{c}\text { Well } \\
\text { Number }\end{array}$ & $\begin{array}{c}\text { West } \\
\text { Longitude }\left({ }^{\circ}\right)\end{array}$ & $\begin{array}{c}\text { North } \\
\text { Latitude }\left({ }^{\circ}\right)\end{array}$ & $\begin{array}{l}\text { Total Depth } \\
\text { (feet) }\end{array}$ & \begin{tabular}{|c|} 
Surface \\
Elevation (feet)
\end{tabular} & $\begin{array}{c}\text { Depth to top of } \\
\text { water interval (feet) }\end{array}$ & $\begin{array}{l}\text { Water interval } \\
\text { thickness (feet) }\end{array}$ & $\begin{array}{c}\text { Geologic unit present } \\
\text { in water interval }\end{array}$ & Down-Hole Lithology of water interval \\
\hline 194 & $7 / 8-23 \mathrm{~L} 1$ & 122.71722 & 38.43743 & 300 & 155 & $\begin{array}{c}48 \\
132 \\
273\end{array}$ & $\begin{array}{l}4 \\
1 \\
5\end{array}$ & $\begin{array}{l}\text { QTge } \\
\text { QTge } \\
\text { QTge }\end{array}$ & $\begin{array}{l}\text { gravel } \\
\text { gravel } \\
\text { gravel }\end{array}$ \\
\hline 195 & $7 / 8-23 \mathrm{M} 1$ & 122.71855 & 38.43698 & 990 & 155 & $\begin{array}{c}54 \\
320 \\
500 \\
751 \\
955\end{array}$ & $\begin{array}{c}66 \\
15 \\
127 \\
63 \\
15\end{array}$ & $\begin{array}{l}\text { QTge(?) } \\
\text { QTge } \\
\text { QTm } \\
\text { QTm } \\
\text { QTm }\end{array}$ & $\begin{array}{l}\text { clay containing streaks of sand and gravel; gravel } \\
\text { sand and gravel } \\
\text { sand and red gravel; sand } \\
\text { sand } \\
\text { sand }\end{array}$ \\
\hline 196 & 7/8-23M2 & 122.71825 & 38.43700 & 210 & 155 & -- & -- & QTge(?) & -- \\
\hline 197 & 7/8-23M3 & 122.71835 & 38.43681 & 300 & 155 & -- & -- & -- & -- \\
\hline 198 & 7/8-23M4 & 122.72208 & 38.43867 & 1279 & 150 & -- & -- & -- & -- \\
\hline 199 & $7 / 8-23 \mathrm{P} 1$ & 122.71585 & 38.43341 & 266 & 150 & $\begin{array}{c}90 \\
116 \\
258\end{array}$ & $\begin{array}{c}5 \\
22 \\
6\end{array}$ & $\begin{array}{l}\text { QTge } \\
\text { QTge } \\
\text { QTge }\end{array}$ & $\begin{array}{l}\text { clay and gravel } \\
\text { clay and gravel; cemented gravel; gravel } \\
\text { clay and gravel }\end{array}$ \\
\hline 200 & 7/8-24A2 & 122.68694 & 38.44516 & 930 & 195 & -- & -- & Qyal, QTge, Tsv(?) & -- \\
\hline 201 & 7/8-24A3 & 122.68688 & 38.44427 & 300 & 195 & -- & -- & Qyal, QTge & -- \\
\hline 202 & 7/8-24A4 & 122.68797 & 38.44467 & 1000 & 195 & -- & -- & Qyal, QTge, Tsv(?) & -- \\
\hline 203 & 7/8-24A5 & 122.68702 & 38.44345 & 291 & 190 & $\begin{array}{c}50 \\
186\end{array}$ & $\begin{array}{l}120 \\
102\end{array}$ & QTge & $\begin{array}{l}-- \\
--\end{array}$ \\
\hline 204 & 7/8-24A6 & 122.68681 & 38.44332 & 1200 & 190 & -- & -- & Qyal(?), QTge, Tsv(?) & -- \\
\hline 205 & 7/8-24K1 & 122.69542 & 38.43724 & 238 & 180 & 234 & 2 & QTge & coarse sand and gravel \\
\hline 206 & $7 / 8-25 \mathrm{~L} 1$ & 122.69874 & 38.42294 & 745 & 175 & $\begin{array}{l}270 \\
545\end{array}$ & $\begin{array}{l}100 \\
200\end{array}$ & QTge & $\begin{array}{l}-- \\
--\end{array}$ \\
\hline 207 & 7/8-25R1 & 122.68935 & 38.41832 & 320 & 235 & 30 & 120 & $\operatorname{Tsv}(?)$ & -- \\
\hline 208 & 7/8-29J1 & 122.76067 & 38.42214 & 700 & 94 & -- & -- & QTge & -- \\
\hline 209 & 7/8-29R1 & 122.76043 & 38.42131 & 682 & 93 & $\begin{array}{l}110 \\
130 \\
150 \\
175 \\
238 \\
650\end{array}$ & $\begin{array}{l}10 \\
15 \\
7 \\
22 \\
15 \\
29\end{array}$ & $\begin{array}{l}\text { QTge } \\
\text { QTge } \\
\text { QTge } \\
\text { QTge } \\
\text { QTge } \\
\text { QTge }\end{array}$ & $\begin{array}{l}\text { cemented gravel } \\
\text { clay, gravel, and boulders } \\
\text { sandy clay and gravel } \\
\text { clay and gravel } \\
\text { cemented gravel } \\
\text { fine free gravel; sandy sediment }\end{array}$ \\
\hline 210 & 7/8-29R2 & 122.76018 & 38.42111 & 801 & 94 & -- & -- & -- & -- \\
\hline 211 & 7/8-30P1 & 122.78891 & 38.41875 & 205 & 85 & -- & -- & QTge, QTm(?) & -- \\
\hline 212 & 7/8-33M1 & 122.75533 & 38.40745 & 452 & 95 & $\begin{array}{c}40 \\
87 \\
146 \\
172 \\
\end{array}$ & $\begin{array}{c}23 \\
17 \\
7 \\
119 \\
\end{array}$ & $\begin{array}{l}\text { QTge } \\
\text { QTge } \\
\text { QTge } \\
\text { QTge }\end{array}$ & $\begin{array}{l}\text { gravel } \\
\text { sand and gravel } \\
\text { sand and gravel } \\
\text { gravel }\end{array}$ \\
\hline
\end{tabular}


Locations and data for water wells of the Santa Rosa Valley, Sonoma County, California

Table 1. Data for selected water wells of the Santa Rosa Valley, Sonoma County, California-Continued

$[--$, no data; *, data for deepened well $]$

\begin{tabular}{|c|c|c|c|c|c|c|c|c|c|}
\hline $\begin{array}{c}\text { Map } \\
\text { Number }\end{array}$ & $\begin{array}{c}\text { Well } \\
\text { Number }\end{array}$ & \begin{tabular}{c|}
$\begin{array}{c}\text { West } \\
\text { Longitude }\left({ }^{\circ}\right)\end{array}$ \\
\end{tabular} & $\begin{array}{c}\text { North } \\
\text { Latitude }\left({ }^{\circ}\right) \\
\end{array}$ & $\begin{array}{c}\text { Total Depth } \\
\text { (feet) }\end{array}$ & \begin{tabular}{c|c} 
Surface \\
Elevation (feet)
\end{tabular} & $\begin{array}{c}\text { Depth to top of } \\
\text { water interval (feet) }\end{array}$ & $\begin{array}{l}\text { Water interval } \\
\text { thickness (feet) }\end{array}$ & \begin{tabular}{|c|}
$\begin{array}{c}\text { Geologic unit present } \\
\text { in water interval }\end{array}$ \\
\end{tabular} & Down-Hole Lithology of water interval \\
\hline & & & & & & $\begin{array}{l}346 \\
422\end{array}$ & $\begin{array}{l}29 \\
30\end{array}$ & $\begin{array}{l}\text { QTge } \\
\text { QTge }\end{array}$ & $\begin{array}{l}\text { sand and gravel } \\
\text { sand and gravel }\end{array}$ \\
\hline 213 & 7/8-35H1 & 122.70504 & 38.41430 & 270 & 150 & -- & -- & QTge & -- \\
\hline 214 & 7/8-36D2 & 122.70329 & 38.41708 & 252 & 165 & 241 & 8 & $\operatorname{Tsv}(?)$ & coarse sand \\
\hline 215 & $7 / 9-2 \mathrm{C} 1$ & 122.82857 & 38.48874 & 610 & 70 & -- & -- & Qyal, Qtge, QTm & -- \\
\hline 216 & 7/9-5N1 & 122.88869 & 38.47668 & 292 & 170 & -- & -- & QTm & -- \\
\hline 217 & 7/9-7G1 & 122.89673 & 38.47189 & 331 & 250 & -- & -- & $\mathrm{KJu}$ & -- \\
\hline 218 & 7/9-8J1 & 122.87304 & 38.46756 & 250 & 215 & 160 & 114 & QTm & sandstone \\
\hline 219 & 7/9-8K1 & 122.87730 & 38.46606 & 350 & 225 & 276 & 74 & QTm & sandstone \\
\hline 220 & 7/9-9F1 & 122.86418 & 38.47045 & 226 & 280 & 186 & 40 & QTm & sand; sandstone \\
\hline 221 & 7/9-10E1 & 122.85014 & 38.46985 & 260 & 200 & 160 & 100 & QTm & sandstone; sand \\
\hline 222 & 7/9-13M1 & 122.81163 & 38.45370 & 320 & 70 & 235 & 57 & QTm & sandy clay \\
\hline 223 & 7/9-13R1 & 122.79843 & 38.44926 & 375 & 70 & $\begin{array}{c}37 \\
70 \\
94 \\
130 \\
265 \\
330\end{array}$ & $\begin{array}{c}9 \\
11 \\
26 \\
40 \\
10 \\
45\end{array}$ & $\begin{array}{l}\text { QTge } \\
\text { QTge } \\
\text { QTge } \\
\text { QTge } \\
\text { QTge } \\
\text { QTge }\end{array}$ & $\begin{array}{l}\text { sand and gravel; clay and gravel } \\
\text { clay and gravel; gravel } \\
\text { clay and gravel } \\
\text { clay and gravel } \\
\text { clay and gravel } \\
\text { sandy clay and gravel; gravel }\end{array}$ \\
\hline 224 & 7/9-14K1 & 122.82137 & 38.45398 & 636 & 80 & $\begin{array}{c}16 \\
86 \\
138\end{array}$ & $\begin{array}{c}24 \\
24 \\
446(136.0)\end{array}$ & $\begin{array}{l}\text { QTge } \\
\text { QTge } \\
\text { QTm }\end{array}$ & $\begin{array}{l}\text { sand; fine gravel; clay } \\
\text { clay, small gravel, and sand } \\
\text { shells and gravel; sand; sand and small gravel; small gravel }\end{array}$ \\
\hline 225 & 7/9-15E2 & 122.84821 & 38.45821 & 220 & 125 & 215 & 5 & QTm & sand and shells \\
\hline 226 & 7/9-15Q1 & 122.84245 & 38.45110 & $200+$ & 75 & -- & -- & QTm & -- \\
\hline 227 & 7/9-16L1 & 122.86211 & 38.45280 & 255 & 220 & -- & -- & QTm & -- \\
\hline 228 & 7/9-16Q1 & 122.85936 & 38.44952 & 321 & 225 & -- & -- & QTm & -- \\
\hline 229 & 7/9-18J1 & 122.89355 & 38.45523 & 650 & 115 & -- & -- & $\mathrm{KJu}$ & -- \\
\hline 230 & 7/9-18K1 & 122.89388 & 38.45338 & 266 & 115 & -- & -- & $\mathrm{KJu}(?)$ & -- \\
\hline 231 & 7/9-21B1 & 122.85976 & 38.44766 & 550 & 240 & -- & -- & QTm & -- \\
\hline 232 & 7/9-21C1 & 122.86412 & 38.44499 & 325 & 225 & 290 & 35 & QTm & sandstone \\
\hline 233 & 7/9-21F1 & 122.86442 & 38.44160 & 320 & 200 & -- & -- & QTm & -- \\
\hline 234 & 7/9-21G1 & 122.85799 & 38.44407 & 530 & 240 & -- & -- & QTm & -- \\
\hline 235 & 7/9-21G2 & 122.85944 & 38.44257 & 240 & 205 & -- & -- & QTm & -- \\
\hline 236 & 7/9-21G3 & 122.86106 & 38.44144 & 408 & 215 & 390 & 18 & QTm & sandstone \\
\hline 237 & 7/9-21J1 & 122.85741 & 38.44006 & 205 & 230 & 195 & 10 & QTm & sandstone \\
\hline 238 & $7 / 9-22 F 1$ & 122.84397 & 38.44204 & 575 & 125 & $\begin{array}{c}42 \\
383 \\
481\end{array}$ & $\begin{array}{l}215 \\
17 \\
31\end{array}$ & $\begin{array}{l}\text { QTm } \\
\text { QTm } \\
\text { QTm }\end{array}$ & $\begin{array}{l}\text { fine sand } \\
\text { small boulders with shells } \\
\text { small boulders with shells }\end{array}$ \\
\hline
\end{tabular}


Locations and data for water wells of the Santa Rosa Valley, Sonoma County, California

Table 1. Data for selected water wells of the Santa Rosa Valley, Sonoma County, California-Continued

$[--$, no data; $*$, data for deepened well]

\begin{tabular}{|c|c|c|c|c|c|c|c|c|c|}
\hline $\begin{array}{l}\text { Map } \\
\text { Number }\end{array}$ & $\begin{array}{c}\text { Well } \\
\text { Number }\end{array}$ & \begin{tabular}{c|} 
West \\
Longitude $\left({ }^{\circ}\right)$
\end{tabular} & $\begin{array}{c}\text { North } \\
\text { Latitude }\left({ }^{\circ}\right) \\
\end{array}$ & $\begin{array}{l}\text { Total Depth } \\
\text { (feet) }\end{array}$ & \begin{tabular}{c|c} 
Surface \\
Elevation (feet)
\end{tabular} & $\begin{array}{c}\text { Depth to top of } \\
\text { water interval (feet) }\end{array}$ & $\begin{array}{l}\text { Water interval } \\
\text { thickness (feet) }\end{array}$ & \begin{tabular}{|c|} 
Geologic unit present \\
in water interval $^{1}$
\end{tabular} & Down-Hole Lithology of water interval \\
\hline & & & & & & 517 & 58 & QTm & fine gravel; coarse gravel \\
\hline 239 & $7 / 9-22 \mathrm{~F} 2$ & 122.84683 & 38.44182 & 380 & 150 & -- & -- & QTm & -- \\
\hline 240 & 7/9-23F1 & 122.82645 & 38.44455 & 610 & 70 & $\begin{array}{l}15 \\
48 \\
70\end{array}$ & $\begin{array}{c}22 \\
22 \\
540\end{array}$ & $\begin{array}{l}\text { QTm } \\
\text { QTm } \\
\text { QTm }\end{array}$ & $\begin{array}{l}\text { sandstone; sand } \\
\text { coarse sandstone; sand; "quicksand" } \\
\text { sandstone and shells; sand and shells; sand and gravel }\end{array}$ \\
\hline 241 & 7/9-23K1 & 122.82298 & 38.43982 & 200 & 85 & -- & -- & QTge, QTm & -- \\
\hline 242 & 7/9-24L2 & 122.80847 & 38.43957 & 330 & 95 & -- & -- & QTge, QTm(?) & -- \\
\hline 243 & 7/9-25A1 & 122.80018 & 38.43081 & 256 & 80 & $\begin{array}{c}90 \\
190\end{array}$ & $\begin{array}{l}16 \\
16\end{array}$ & $\begin{array}{l}\text { QTm } \\
\text { QTm }\end{array}$ & $\begin{array}{l}\text { sand } \\
\text { sand }\end{array}$ \\
\hline 244 & 7/9-26M1 & 122.83320 & 38.42598 & 206 & 80 & -- & -- & QTm & -- \\
\hline 245 & 7/9-27E1 & 122.84975 & 38.42645 & 260 & 145 & 12 & 248 & QTm & sandstone and clam shells; sand and gravel; sand \\
\hline 246 & 7/9-27E2 & 122.84868 & 38.42894 & 370 & 165 & $\begin{array}{c}0 \\
90\end{array}$ & $\begin{array}{c}47 \\
280\end{array}$ & $\begin{array}{l}\text { QTm } \\
\text { QTm }\end{array}$ & $\begin{array}{l}\text { sand and gravel; quicksand } \\
\text { sandstone; gravel and sand }\end{array}$ \\
\hline 247 & 7/9-27F1 & 122.84718 & 38.42763 & 200 & 150 & -- & -- & QTm & -- \\
\hline 248 & 7/9-28G1 & 122.85955 & 38.42745 & 260 & 120 & 90 & 170 & QTm & sandstone \\
\hline 249 & 7/9-28G2 & 122.85927 & 38.42723 & 240 & 115 & 40 & 200 & QTm & sandstone; sandstone and clam shells \\
\hline 250 & 7/9-28L1 & 122.86415 & 38.42427 & 565 & 110 & 5 & 537 & QTm & sandstone (20' of surface casing) \\
\hline 251 & 7/9-28P1 & 122.86626 & 38.41935 & 585 & 115 & -- & -- & QTm & -- \\
\hline 252 & 7/9-29J1 & 122.87244 & 38.42386 & 400 & 120 & -- & -- & QTm & -- \\
\hline 253 & 7/9-29J2 & 122.87276 & 38.42487 & 657 & 120 & $\begin{array}{c}46 \\
548\end{array}$ & $\begin{array}{l}453 \\
109\end{array}$ & $\begin{array}{l}\text { QTm } \\
\text { QTm }\end{array}$ & $\begin{array}{l}\text { sandstone } \\
\text { soft sandstone; sand with shells }\end{array}$ \\
\hline 254 & 7/9-30A1 & 122.89096 & 38.43375 & 260 & 135 & -- & -- & QTm & -- \\
\hline 255 & 7/9-30G2 & 122.89643 & 38.42929 & 200 & 200 & -- & -- & QTm & -- \\
\hline 256 & 7/9-30J1 & 122.89033 & 38.42637 & 200 & 285 & 160 & 40 & QTm & sandstone \\
\hline 257 & 7/9-31A1 & 122.88965 & 38.41606 & 240 & 410 & -- & -- & QTm & -- \\
\hline 258 & 7/9-31J1 & 122.89202 & 38.40979 & 352 & 465 & $\begin{array}{c}40 \\
185 \\
296\end{array}$ & $\begin{array}{l}22 \\
56 \\
56\end{array}$ & $\begin{array}{l}\text { QTm } \\
\text { QTm } \\
\text { QTm }\end{array}$ & $\begin{array}{l}\text { fine sand } \\
\text { sandstone with clam shells } \\
\text { sandstone containing streak gravel }\end{array}$ \\
\hline 259 & 7/9-31Q1 & 122.89345 & 38.40751 & 235 & 480 & 90 & 145 & QTm & sandstone \\
\hline 260 & 7/9-31Q3 & 122.89419 & 38.40611 & 250 & 470 & -- & -- & QTm & -- \\
\hline 261 & 7/9-32E1 & 122.88781 & 38.41252 & 200 & 485 & -- & -- & QTm & -- \\
\hline 262 & $7 / 9-32 \mathrm{~F} 2$ & 122.87994 & 38.41407 & 365 & 395 & -- & -- & QTm & -- \\
\hline 263 & 7/9-33A1 & 122.85343 & 38.41802 & 245 & 225 & -- & -- & QTm & -- \\
\hline 264 & 7/9-33D2 & 122.86749 & 38.41824 & 300 & 155 & -- & -- & QTm & -- \\
\hline 265 & 7/9-33F1 & 122.86576 & 38.41278 & 200 & 165 & 0 & 200 & QTm & sand; sandstone \\
\hline 266 & 7/9-34G1 & 122.84006 & 38.41451 & 358 & 195 & 349 & 9 & QTm & coarse soft sandstone \\
\hline 267 & 7/9-34J1 & 122.83581 & 38.40961 & 230 & 190 & -- & -- & QTm & -- \\
\hline
\end{tabular}


Locations and data for water wells of the Santa Rosa Valley, Sonoma County, California

[--, no data; *, data for deepened well]

\begin{tabular}{|c|c|c|c|c|c|c|c|c|c|}
\hline $\begin{array}{c}\text { Map } \\
\text { Number }\end{array}$ & $\begin{array}{c}\text { Well } \\
\text { Number }\end{array}$ & $\begin{array}{c}\text { West } \\
\text { Longitude }\left({ }^{\circ}\right) \\
\end{array}$ & $\begin{array}{c}\text { North } \\
\text { Latitude }\left({ }^{\circ}\right) \\
\end{array}$ & $\begin{array}{c}\text { Total Depth } \\
\text { (feet) }\end{array}$ & $\begin{array}{c}\text { Surface } \\
\text { Elevation (feet) }\end{array}$ & $\begin{array}{c}\text { Depth to top of } \\
\text { water interval (feet) }\end{array}$ & $\begin{array}{l}\text { Water interval } \\
\text { thickness (feet) }\end{array}$ & $\begin{array}{c}\text { Geologic unit present } \\
\text { in water interval }^{1}\end{array}$ & Down-Hole Lithology of water interval \\
\hline 268 & $7 / 9-34 J 2$ & 122.83490 & 38.40884 & 350 & 310 & 310 & 20 & QTm & -- \\
\hline 269 & 7/9-34L1 & 122.84444 & 38.41104 & 315 & 300 & 300 & 15 & QTm & sand \\
\hline 270 & 7/9-35E1 & 122.83110 & 38.41273 & 253 & 150 & -- & -- & QTm & -- \\
\hline 271 & 7/9-36F1 & 122.80650 & 38.41312 & 270 & 85 & $\begin{array}{c}40 \\
60 \\
160 \\
200\end{array}$ & $\begin{array}{c}5 \\
90 \\
18 \\
70\end{array}$ & $\begin{array}{l}\text { QTge } \\
\text { QTge } \\
\text { QTm } \\
\text { QTm }\end{array}$ & $\begin{array}{l}\text { pea gravel } \\
\text { fine gravel; large gravel } \\
\text { river sand } \\
\text { fine and coarse gravel; quicksand }\end{array}$ \\
\hline 272 & 7/9-36H1 & 122.80048 & 38.41485 & 568 & 85 & $\begin{array}{c}70 \\
280\end{array}$ & $\begin{array}{c}32 \\
230\end{array}$ & $\begin{array}{l}\text { QTge } \\
\text { QTm }\end{array}$ & $\begin{array}{l}\text { gravel } \\
\text { sandstone; gravel and sand; sand }\end{array}$ \\
\hline 273 & 8/8-17L1 & 122.77222 & 38.54059 & 278 & 185 & -- & -- & QTge & -- \\
\hline 274 & 8/8-19M1 & 122.79322 & 38.52553 & 260 & 105 & $\begin{array}{l}27 \\
115 \\
160 \\
205\end{array}$ & $\begin{array}{l}18 \\
15 \\
25 \\
55\end{array}$ & $\begin{array}{l}\text { QTge } \\
\text { QTge } \\
\text { QTge } \\
\text { QTge }\end{array}$ & $\begin{array}{l}\text { sand and fine gravel; coarse packed gravel } \\
\text { small boulders } \\
\text { fine gravel and sand } \\
\text { coarse packed gravel; medium gravel }\end{array}$ \\
\hline 275 & 8/8-19N1 & 122.79435 & 38.52263 & 249 & 105 & $\begin{array}{c}27 \\
105 \\
150 \\
200\end{array}$ & $\begin{array}{l}18 \\
15 \\
25 \\
49\end{array}$ & $\begin{array}{l}\text { QTge } \\
\text { QTge } \\
\text { QTge } \\
\text { QTge }\end{array}$ & $\begin{array}{l}\text { packed fine sandy gravel; coarse packed gravel } \\
\text { "small bouldery" sand } \\
\text { fine gravel and coarse sand } \\
\text { packed coarse gravelly sand }\end{array}$ \\
\hline 276 & $8 / 8-20 \mathrm{~B} 2$ & 122.76697 & 38.53308 & 231 & 185 & 202 & 25 & QTge & sand clay and gravel; gravel \\
\hline 277 & 8/8-20D1 & 122.77436 & 38.53123 & 260 & 145 & -- & -- & QTge & -- \\
\hline 278 & 8/8-20Q1 & 122.76602 & 38.52081 & 310 & 140 & 20 & 275 & QTge & gravel and sand; gravel and some clay; gravel \\
\hline 279 & 8/8-30L1 & 122.78918 & 38.51149 & 463 & 120 & $\begin{array}{c}90 \\
204\end{array}$ & $\begin{array}{c}98 \\
259\end{array}$ & $\begin{array}{l}\text { QTge } \\
\text { QTge }\end{array}$ & $\begin{array}{l}\text { clay and gravel; gravel } \\
\text { sandy clay and gravel; sandy clay }\end{array}$ \\
\hline 280 & 8/8-30N1 & 122.79296 & 38.50756 & 455 & 120 & $\begin{array}{c}60 \\
119 \\
231 \\
253 \\
405\end{array}$ & $\begin{array}{c}38 \\
104 \\
8 \\
97 \\
45\end{array}$ & $\begin{array}{l}\text { QTge } \\
\text { QTge } \\
\text { QTge } \\
\text { QTge } \\
\text { QTge }\end{array}$ & $\begin{array}{l}\text { clay and gravel } \\
\text { clay and gravel; gravel } \\
\text { cemented gravel } \\
\text { sandy clay and gravel } \\
\text { sandy clay and gravel }\end{array}$ \\
\hline 281 & $8 / 8-30 \mathrm{~N} 2$ & 122.79556 & 38.50759 & 600 & 120 & $\begin{array}{c}62 \\
190 \\
425 \\
580\end{array}$ & $\begin{array}{c}121 \\
200 \\
11 \\
5\end{array}$ & $\begin{array}{l}\text { QTge } \\
\text { QTge } \\
\text { QTge } \\
\text { QTge }\end{array}$ & $\begin{array}{l}\text { clay and gravel; gravel } \\
\text { clay and gravel; sandy clay and gravel } \\
\text { sandy clay; clay and gravel } \\
\text { clay and gravel }\end{array}$ \\
\hline 282 & 8/8-31B1 & 122.78613 & 38.50357 & 210 & 115 & 188 & 18 & QTge & gravel; clay and gravel \\
\hline 283 & 8/8-34Q1 & 122.73067 & 38.49123 & 285 & 345 & -- & -- & QTge & -- \\
\hline 284 & 8/9-1M1 & 122.81177 & 38.57031 & 315 & 185 & $\begin{array}{l}250 \\
261 \\
281\end{array}$ & $\begin{array}{l}2 \\
1 \\
1\end{array}$ & $\begin{array}{l}\text { QTge } \\
\text { QTge } \\
\text { QTge }\end{array}$ & $\begin{array}{l}\text { sand and gravel } \\
\text { sand and gravel } \\
\text { sand and gravel }\end{array}$ \\
\hline
\end{tabular}


Locations and data for water wells of the Santa Rosa Valley, Sonoma County, California

by Zenon C. Valin and Robert J. McLaughlin

Table 1. Data for selected water wells of the Santa Rosa Valley, Sonoma County, California-Continued

$[--$, no data; *, data for deepened well]

\begin{tabular}{|c|c|c|c|c|c|c|c|c|c|}
\hline $\begin{array}{c}\text { Map } \\
\text { Number }\end{array}$ & $\begin{array}{c}\text { Well } \\
\text { Number }\end{array}$ & \begin{tabular}{|c|} 
West \\
Longitude $\left({ }^{\circ}\right)$ \\
\end{tabular} & $\begin{array}{c}\text { North } \\
\text { Latitude }\left({ }^{\circ}\right) \\
\end{array}$ & $\begin{array}{l}\text { Total Depth } \\
\text { (feet) }\end{array}$ & \begin{tabular}{|c|} 
Surface \\
Elevation (feet)
\end{tabular} & $\begin{array}{c}\text { Depth to top of } \\
\text { water interval (feet) }\end{array}$ & $\begin{array}{l}\text { Water interval } \\
\text { thickness (feet) }\end{array}$ & \begin{tabular}{|c|} 
Geologic unit present \\
in water interval $^{1}$
\end{tabular} & Down-Hole Lithology of water interval \\
\hline & & & & & & 298 & 2 & QTge & sand and gravel \\
\hline 285 & 8/9-10R1 & 122.83569 & 38.55077 & 400 & 165 & -- & -- & QTge & -- \\
\hline 286 & $8 / 9-13 \mathrm{C} 1$ & 122.80721 & 38.54531 & 202 & 105 & 65 & 137 & QTge & clay and gravel; gravel \\
\hline 287 & $8 / 9-13 N 1$ & 122.81207 & 38.53715 & 208 & 90 & $\begin{array}{c}14 \\
70 \\
82 \\
98 \\
130 \\
152\end{array}$ & $\begin{array}{c}26 \\
4 \\
4 \\
12 \\
6 \\
32\end{array}$ & $\begin{array}{l}\text { QTge } \\
\text { QTge } \\
\text { QTge } \\
\text { QTge } \\
\text { QTge } \\
\text { QTge }\end{array}$ & $\begin{array}{l}\text { gravel } \\
\text { gravel } \\
\text { gravel } \\
\text { sand and gravel } \\
\text { sand, clay, and gravel } \\
\text { gravel }\end{array}$ \\
\hline 288 & 8/9-14L1 & 122.82986 & 38.53980 & 265 & 95 & $\begin{array}{c}53 \\
83 \\
106 \\
242\end{array}$ & $\begin{array}{c}5 \\
6 \\
3 \\
11\end{array}$ & $\begin{array}{l}\text { QTge } \\
\text { QTge } \\
\text { QTge } \\
\text { QTge }\end{array}$ & $\begin{array}{l}\text { loose gravel } \\
\text { cemented gravel } \\
\text { loose gravel } \\
\text { pea gravel }\end{array}$ \\
\hline 289 & 8/9-14P1 & 122.82924 & 38.53583 & 264 & 95 & $\begin{array}{c}11 \\
19 \\
60 \\
118 \\
176 \\
253\end{array}$ & $\begin{array}{c}5 \\
18 \\
5 \\
2 \\
8 \\
11\end{array}$ & $\begin{array}{l}\text { QTge } \\
\text { QTge } \\
\text { QTge } \\
\text { QTge } \\
\text { QTge } \\
\text { QTge }\end{array}$ & $\begin{array}{l}\text { sand and gravel } \\
\text { large gravel } \\
\text { sand } \\
\text { gravel } \\
\text { fine gravel } \\
\text { pea gravel }\end{array}$ \\
\hline 290 & $8 / 9-23 \mathrm{G} 1$ & 122.82274 & 38.53074 & 225 & 75 & $\begin{array}{c}18 \\
37 \\
79 \\
128 \\
200\end{array}$ & $\begin{array}{l}10 \\
15 \\
16 \\
10 \\
23\end{array}$ & $\begin{array}{l}\text { QTge } \\
\text { QTge } \\
\text { QTge } \\
\text { QTge } \\
\text { QTge }\end{array}$ & $\begin{array}{l}\text { gravel } \\
\text { sand; pea gravel } \\
\text { fine gravel } \\
\text { sand and gravel } \\
\text { gravel }\end{array}$ \\
\hline 290 & 8/9-23G1* & 122.82274 & 38.53074 & 440 & 75 & 276 & 4 & QTge & gravel \\
\hline 291 & 8/9-23L1 & 122.82721 & 38.52734 & 429 & 75 & $\begin{array}{c}15 \\
56 \\
74 \\
306 \\
376\end{array}$ & $\begin{array}{l}36 \\
14 \\
11 \\
45 \\
27\end{array}$ & $\begin{array}{l}\text { QTge } \\
\text { QTge } \\
\text { QTge } \\
\text { QTge } \\
\text { QTge }\end{array}$ & $\begin{array}{l}\text { coarse gravel; sand } \\
\text { coarse gravel } \\
\text { coarse gravel } \\
\text { gravel } \\
\text { coarse sand and washed sand }\end{array}$ \\
\hline 292 & 8/9-24E1 & 122.81148 & 38.52995 & 330 & 80 & -- & -- & Qyal(?), QTge & -- \\
\hline 293 & 8/9-26D1 & 122.83206 & 38.51907 & 464 & 75 & $\begin{array}{l}240 \\
394\end{array}$ & $\begin{array}{l}40 \\
68\end{array}$ & $\begin{array}{l}\text { QTge } \\
\text { QTge }\end{array}$ & $\begin{array}{l}\text { sandy clay and gravel; gravel } \\
\text { clay and gravel; gravel }\end{array}$ \\
\hline 294 & $8 / 9-27 \mathrm{~K} 1$ & 122.83936 & 38.51137 & 333 & 60 & $\begin{array}{c}50 \\
303\end{array}$ & $\begin{array}{c}2 \\
30\end{array}$ & $\begin{array}{l}\text { QTge } \\
\text { QTm }\end{array}$ & $\begin{array}{l}\text { sand and gravel } \\
\text { gravel and clam shells }\end{array}$ \\
\hline 295 & 8/9-33G1 & 122.85868 & 38.49966 & 358 & 100 & -- & -- & -- & -- \\
\hline 296 & 8/9-33J1 & 122.85358 & 38.49793 & 325 & 45 & -- & -- & -- & -- \\
\hline
\end{tabular}


Locations and data for water wells of the Santa Rosa Valley, Sonoma County, California

Table 1. Data for selected water wells of the Santa Rosa Vallev, Sonoma County, California-Continued

$[--$, no data; *, data for deepened well]

\begin{tabular}{|c|c|c|c|c|c|c|c|c|c|}
\hline $\begin{array}{l}\text { Map } \\
\text { Number }\end{array}$ & $\begin{array}{c}\text { Well } \\
\text { Number }\end{array}$ & $\begin{array}{c}\text { West } \\
\text { Longitude }\left({ }^{\circ}\right)\end{array}$ & $\begin{array}{c}\text { North } \\
\text { Latitude }\left({ }^{\circ}\right)\end{array}$ & $\begin{array}{l}\text { Total Depth } \\
\text { (feet) }\end{array}$ & \begin{tabular}{|c|} 
Surface \\
Elevation (feet)
\end{tabular} & $\begin{array}{c}\text { Depth to top of } \\
\text { water interval (feet) }\end{array}$ & $\begin{array}{l}\text { Water interval } \\
\text { thickness (feet) }\end{array}$ & \begin{tabular}{|c|} 
Geologic unit present \\
in water interval
\end{tabular} & Down-Hole Lithology of water interval \\
\hline 297 & 8/9-34R1 & 122.83441 & 38.49432 & 360 & 65 & -- & -- & Qyal, QTge, QTm & -- \\
\hline 298 & 8/9-34R2 & 122.83444 & 38.49123 & 490 & 65 & $\begin{array}{c}20 \\
118 \\
190\end{array}$ & $\begin{array}{c}50 \\
41 \\
160\end{array}$ & $\begin{array}{l}\text { Qyal } \\
\text { QTge } \\
\text { QTm }\end{array}$ & $\begin{array}{l}\text { pea gravel; sand } \\
\text { clay and gravel; small gravel } \\
\text { small gravel; sand; shell and gravel; shells }\end{array}$ \\
\hline 299 & $8 / 9-35 \mathrm{~K} 1$ & 122.82190 & 38.49616 & 498 & 80 & $\begin{array}{c}50 \\
198 \\
268 \\
398 \\
465\end{array}$ & $\begin{array}{r}22 \\
10 \\
52 \\
27 \\
--\end{array}$ & $\begin{array}{c}\text { QTge } \\
\text { QTge } \\
\text { QTge, QTm } \\
\text { QTge, QTm } \\
\text { QTge, QTm }\end{array}$ & $\begin{array}{l}\text { cemented gravel } \\
\text { cemented gravel } \\
\text { sandstone; clay and gravel } \\
\text { sandstone; cemented gravel } \\
\text { sandstone; cemented gravel; sand and gravel }\end{array}$ \\
\hline 300 & 8/9-35M1 & 122.83326 & 38.49475 & 475 & 65 & -- & -- & Qyal, QTge, QTm & -- \\
\hline 301 & 8/9-36G1 & 122.80103 & 38.49917 & 642 & 100 & -- & -- & QTge, QTm & -- \\
\hline 302 & 8/9-36K1 & 122.80515 & 38.49665 & 1325 & 95 & -- & -- & -- & -- \\
\hline 303 & 8/9-36L1 & 122.80878 & 38.49755 & 565 & 95 & -- & -- & QTge & -- \\
\hline 304 & 8/9-36P1 & 122.80970 & 38.49241 & 1048 & 90 & -- & -- & QTge, QTm & -- \\
\hline
\end{tabular}

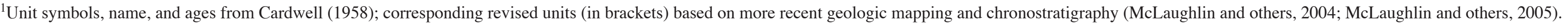

Qyal, Younger alluvium (Recent) [Quaternary alluvium (Holocene and Pleistocene)]

Qoal, Terrace deposits and older alluvium (Pleistocene) [Quaternary alluvium (Holocene and Pleistocene)]

QTge, Glen Ellen Formation (Pliocene (?) and Pleistocene) [Nonmarine sedimentary rocks (Pleistocene and Pliocene)]

QTm, Merced Formation (Pliocene and Pleistocene (?)) [Marine sedimentary rocks (Pliocene and late Miocene)]

Tp, Petaluma Formation (Pliocene) [Intercalated marine and nonmarine sedimentary rocks (Pliocene and late Miocene)

Tsv, Sonoma Volcanics (Pliocene) [Volcanic rocks (Tertiary)]

KJu, Cretaceous and Jurassic rocks, undifferentiated [Basement rocks (pre-Tertiary)]

\section{REFERENCES CITED}

Cardwell, G.T., 1958, Geology and Ground Water in the Santa Rosa and Petaluma Valley areas, Sonoma County, California: U.S. Geological Survey Water-Supply Paper 1427, 273 p.

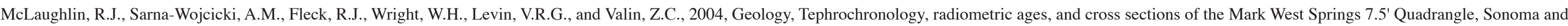
Napa Counties, California: U.S. Geological Survey Miscellaneous Field Studies Map MF2858, scale 1:24,000, 2 sheets. http//pubs.usgs.gov/sim/2004/2858/

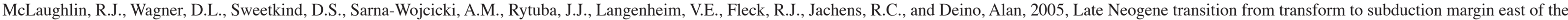
San Andreas Fault in the wine country of the northern San Francisco Bay Area, California: Fieldtrip guidebook and volume for the Joint Meeting of the Cordilleran Section GSA and Pacific Section AAPG, 112 p.

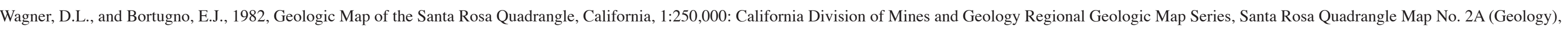
Sheet 1 of 5 . 